\title{
A trans-complementation system for SARS-CoV-2
}

2 Xianwen Zhang ${ }^{1,11}$, Yang Liu ${ }^{1,11}$, Jianying Liü ${ }^{2,3}$, Adam L. Bailey ${ }^{4}$, Kenneth S. Plante ${ }^{2,3,5}$, Jessica 3 A. Plante ${ }^{2,3,5}$, Jing Zou ${ }^{1}$, Hongjie Xia ${ }^{1}$, Nathen Bopp ${ }^{6}$, Patricia Aguilar $^{6}$, Ping Ren ${ }^{3},{ }^{6}$, Vineet D. $4 \quad$ Menachery ${ }^{2,3}$, Michael S. Diamond ${ }^{4,7,8}$, Scott C. Weaver ${ }^{2,3,5,9}$, Xuping Xie $^{1,3^{*}}$, Pei-Yong Shi ${ }^{1,3,9,10,12^{*}}$

$7{ }^{1}$ Department of Biochemistry and Molecular Biology, University of Texas Medical Branch,

8 Galveston TX, USA

$9{ }^{2}$ Department of Microbiology and Immunology, University of Texas Medical Branch, Galveston 10 TX, USA

$11{ }^{3}$ Institute for Human Infections and Immunity, University of Texas Medical Branch, Galveston, 12 TX, USA

$13{ }^{4}$ Department of Pathology \& Immunology, Washington University School of Medicine, St. Louis, $14 \mathrm{MO}$, USA

$15{ }^{5}$ World Reference Center for Emerging Viruses and Arboviruses, University of Texas Medical

16 Branch, Galveston, TX, USA

$17{ }^{6}$ Department of Pathology, University of Texas Medical Branch, Galveston TX, USA

$18{ }^{7}$ Department of Medicine, Washington University School of Medicine, St. Louis, MO, USA

$19{ }^{8}$ Department of Molecular Microbiology, Washington University School of Medicine, St. Louis,

$20 \mathrm{MO}$, USA

$21{ }^{9}$ Sealy Institute for Vaccine Sciences, University of Texas Medical Branch, Galveston, TX, USA

$22{ }^{10}$ Sealy Center for Structural Biology \& Molecular Biophysics, University of Texas Medical

23 Branch, Galveston, TX, USA

$24 \quad{ }^{11}$ These authors contributed equally

$25{ }^{12}$ Lead Contact: Pei-Yong Shi

*Correspondence: X.X. (xuxie@UTMB.edu) or P.-Y.S. (peshi@UTMB.edu)

27 Keywords: SARS-CoV-2, COVID-19, coronavirus, diagnosis, vaccine, antiviral 


\section{ABSTRACT}

The biosafety level-3 (BSL-3) requirement to culture severe acute respiratory syndrome

30 coronavirus 2 (SARS-CoV-2) is a bottleneck for research and countermeasure development.

31 Here we report a trans-complementation system that produces single-round infectious SARS-

32 CoV-2 that recapitulates authentic viral replication. We demonstrate that the single-round

33 infectious SARS-CoV-2 can be used at BSL-2 laboratories for high-throughput neutralization

34 and antiviral testing. The trans-complementation system consists of two components: a genomic

35 viral RNA containing a deletion of ORF3 and envelope gene, and a producer cell line expressing

36 the two deleted genes. Trans-complementation of the two components generates virions that

37 can infect naive cells for only one round, but does not produce wild-type SARS-CoV-2.

38 Hamsters and K18-hACE2 transgenic mice inoculated with the complementation-derived virions

39 exhibited no detectable disease, even after intracranial inoculation with the highest possible

40 dose. The results suggest that the trans-complementation platform can be safely used at BSL-2

41 laboratories for research and countermeasure development.

\section{INTRODUCTION}

44 Three zoonotic betacoronaviruses have emerged to cause global epidemics or

45 pandemics in less than twenty years: severe acute respiratory syndrome coronavirus (SARS-

$46 \mathrm{CoV}$ ) in 2002, Middle East respiratory syndrome coronavirus (MERS-CoV) in 2012, and SARS-

47 CoV-2 in 2019 (1). The coronavirus disease 2019 (COVID-19) pandemic has caused

48 unprecedented social and economic disruption. As of January 16, 2021, SARS-CoV-2 had

49 infected over 94 million people, leading to over 2 million deaths

50 (https://www.worldometers.info/coronavirus/). In response to the pandemic, the scientific

51 community has rapidly developed experimental platforms to study COVID-19 and to develop

52 countermeasures. Several groups have established infectious cDNA clones and reporter SARS- 
53 CoV-2 to facilitate the development and analysis of first-generation vaccines and therapeutics

54 (2-6). However, since SARS-CoV-2 is a biosafety level-3 (BSL-3) pathogen, the requirement of

55 high containment represents a bottleneck for antiviral and vaccine evaluation. Thus, a BSL-2

56 cell culture system that recapitulates authentic viral replication is urgently needed.

The genome of SARS-CoV-2 is a positive-sense, single-stranded RNA of approximately

$5830 \mathrm{~kb}$ in length. The SARS-CoV-2 virion consists of an internal nucleocapsid [formed by the

59 genomic RNA coated with nucleocapsid (N) proteins] and an external envelope [formed by a

60 cell-derived bilipid membrane embedded with spike (S), membrane (M), and envelope (E)

61 proteins] (7). The genomic RNA encodes open-reading-frames (ORFs) for replicase

62 (ORF1a/ORF1b), S, E, M, and N proteins, as well as seven additional ORFs for accessory

63 proteins (1). Stable cell lines containing replicons (self-replicating viral RNA genomes with one

64 or more gene deletions) have been developed for many viruses, including coronaviruses (8-12).

65 Because replicons lack structural genes, they are not infectious and can safely be manipulated

66 in BSL-2 laboratories. For SARS-CoV-2, although a transient replicon system has been

67 established (13), no stable replicon cell line has been reported. To overcome this gap, we have

68 developed a single-round infectious SARS-CoV-2 through trans-complementation. The single-

69 round SARS-CoV-2 is engineered with a reporter gene that facilitates high-throughput antiviral

70 screening and neutralizing antibody measurement. We validated the safety of the system in cell

71 cultures, hamsters, and highly susceptible human angiotensin-converting enzyme 2 (hACE2)

72 transgenic mice. Our results suggest that the trans-complementation system can be used safely

73 at BSL-2 laboratories.

\section{RESULTS}

A single-round infectious SARS-CoV-2 system. Fig. 1A depicts the trans-

77 complementation system to produce single-round infectious SARS-CoV-2. The system contains 
78 two components: (i) a viral RNA containing a mNeonGreen (mNG) reporter gene and a deletion

79 of ORF3 and E genes ( $\triangle$ ORF3-E; Fig. 1B) and (ii) a Vero E6 cell line expressing the ORF3 and

80 E proteins under a doxycycline inducible promoter (Vero-ORF3-E; Fig. 1C-D). Upon

81 electroporation of $\triangle$ ORF3-E RNA into Vero-ORF3-E cells and addition of doxycycline, trans-

82 complementation enables production of virions that can continuously infect and amplify on Vero-

83 ORF3-E cells; however, these virions can only infect normal cells for a single round due to the

84 lack of ORF3 and E genes in the packaged RNA genome (Fig. 1A).

85 Our trans-complementation system is engineered with several safeguards to eliminate 86 wild-type (WT) SARS-CoV-2 production. Besides the ORF3-E deletion, the $\triangle$ ORF3-E viral RNA

87 contained two additional modifications. (i) The transcription regulatory sequence (TRS) of

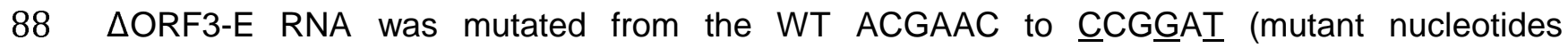

89 underlined; Fig. 1B). Recombination between the TRS-mutated $\triangle O R F 3-E$ RNA with

90 inadvertently contaminating viral RNA would therefore not produce replicative virus $(14,15)$. (ii)

91 An mNG gene was engineered at ORF7 of $\triangle O R F 3-E$ RNA to facilitate the detection of viral

92 replication (Fig. 1B). The trans-complementing Vero-ORF3-E cell lines were produced by

93 transducing Vero E6 cells with a lentivirus encoding the following elements (Fig. S1A): a

94 TRE3GS promoter that allows doxycycline to induce ORF3 and E protein expression (Fig. 1C-D

95 and S1B); an mCherry gene that facilitates selection of cell lines with high levels of protein

96 expression (Fig. S1C); a foot-and-mouth disease virus 2A (FMDV 2A) autocleavage site that

97 enables translation of individual mCherry and viral E protein; and an encephalomyocarditis virus

98 internal ribosomal entry site (EMCV IRES) that bicistronically translates the ORF3 protein. The

99 above design eliminated overlapping sequences between the ORF3-E mRNA and $\triangle \mathrm{ORF3-E}$

100 viral RNA, thus minimizing homologous recombination during trans-complementation. The Vero-

101 ORF3-E cell line stably expressed the engineered proteins after 20 rounds of passaging, as 102 indicated by the mCherry reporter (Fig. 1D). 
104 produced virions of $\sim 10^{4}$ median Tissue Culture Infectious Dose $\left(\mathrm{TCID}_{50}\right) / \mathrm{ml}$ titer (Fig. 1E). The $105 \triangle$ ORF3-E mNG virion exhibited a diameter of $91 \mathrm{~nm}$ under negative staining electron 106 microscopy (Fig. 1F). The $\triangle$ ORF3-E mNG virion produced in the supernatant could infect Vero107 ORF3-E cells for multiple rounds, but for only one round on naïve Vero E6 (Fig. 1G-H), Calu-3, 108 or hACE2-expressing A549 cells (A549-hACE2; Fig. S2). As controls, WT mNG SARS-CoV-2 109 could infect cells for multiple rounds (Fig. S2). These results indicate that the trans110 complementation system produces virions that can only infect WT cells for single round.

111 Adaptive mutations to improve virion production. To improve the efficiency of the

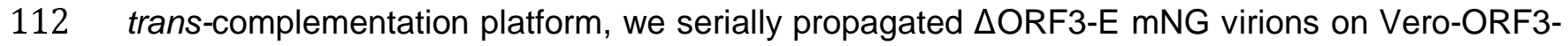

113 E cells for 10 passages (3-4 days per passage) to select for adaptive mutations. The P10 virion 114 replicated to higher titers than the P1 virion on Vero-ORF3-E cells (Fig. 2A), retained the mNG 115 reporter (Fig. 2B-C), and still infected normal Vero cells for only single round (Fig. S3). Whole 116 genome sequencing of the P10 virion revealed three mutations in the nsp1, nsp4, and S genes 117 (Fig. 2D). Engineering of these mutations into $\triangle$ ORF3-E mNG RNA showed that all three were 118 required to enhance the trans-complementation efficiency, producing $10^{6} \mathrm{TCID}_{50} / \mathrm{ml}$ of virions 119 (Fig. 2A-B). These results indicate that (i) adaptive mutations can be selected to improve the 120 yield of single-round virions and (ii) WT virus is not produced from the trans-complementation 121 system.

122 Exclusion of WT SARS-CoV-2 production. To confirm that no WT SARS-CoV-2 is 123 inadvertently produced during trans-complementation, we performed four additional selections

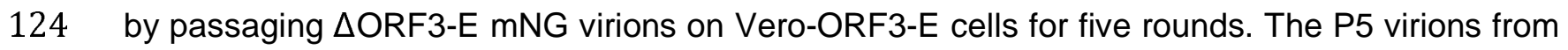
125 selections I-III could only infect Vero cells for single round (Fig. S4A-B). Unexpectedly, 126 selection IV produced P5 (S-IV-P5) virions that could infect parental Vero E6 cells for more than 127 one round, though at a barely detectable level of $\sim 10^{2} \mathrm{TCID}_{50} / \mathrm{ml}$, which was $>100,000$-fold 
128 lower than the WT mNG SARS-CoV-2 titers (Fig. S4C). To remove the single-round virion from

129 the multi-round virion in the S-IV-P5 stock, we passaged the S-IV-P5 virion stock on Vero E6

130 cells for two rounds, resulting in S-IV-P5-Vero-P2 virion capable of multi-round infection. Full-

131 genome sequencing revealed that the S-IV-P5-Vero-P2 virion retained the ORF3-E deletion but

132 accumulated mutations in nsp15, nsp16, $S$, and $M$ genes (Fig. S4D). Engineering the

133 accumulated mutations into $\triangle$ ORF3-E mNG RNA showed that the M mutation T130N conferred

134 multi-round infection on Vero cells (Fig. S4E). Residue T130 is predicted to be on the intra-

135 virion side of the M protein $(16,17)$, and is conserved in SARS-CoV and SARS-CoV-2 (Fig.

136 S4F). The results indicate that, despite an absence of WT SARS-CoV-2 production and a lack

137 of ORF and E genes, the trans-complementation system could produce mutant virions capable

138 of infecting parental Vero cells for multiple rounds at a barely detectable level.

139 Next, we continuously cultured the S-IV-P5 variant on parental Vero E6 cells for 10

140 rounds (3-4 days per round) to select for potential virions with improved replication efficiency.

141 However, passage did not improve viral replication on Vero cells (Fig. S5). The result suggests

142 that, due to the lack of ORF3 and E gene, the S-IV-P5 virion is unlikely to gain efficient multiple-

143 round amplification on normal cells through adaptation.

144 Safety evaluation of $\Delta$ ORF3-E virions in vivo. We examined the virulence of $\Delta$ ORF3-

$145 \mathrm{E}$ mNG virion in hamsters and K18-hACE2 transgenic mice (18-20). After intranasal inoculation

146 with $6 \times 10^{5} \mathrm{TCID}_{50}$ of $\triangle \mathrm{ORF3}-\mathrm{E} \mathrm{mNG}$ virion (the highest possible infecting dose; Fig. 3A),

147 hamsters did not lose weight (Fig. 3B) or develop detectable disease (Fig. 3C). In contrast, $10^{5}$

$148 \mathrm{TCID}_{50}$ of WT SARS-CoV-2-infected hamsters developed weight loss and mild disease (e.g.,

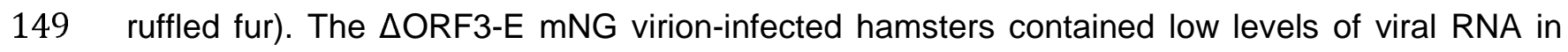

150 nasal washes (Fig. 3D) and oral swabs (Fig. 3E). Viral RNA levels in the trachea and lungs

151 from the $\triangle$ ORF3-E virion-infected animals were 5,000- and 400-fold lower than those from the

152 WT virus-infected hamsters, respectively (Fig. 3F). Next, we examined the S-IV-P5-Vero-P2 
153 virion, capable of infecting Vero cells for multiple rounds, in hamsters. To maximize the infection

154 dose of S-IV-P5-Vero-P2 virion, we amplified S-IV-P5-Vero-P2 on Vero-ORF3-E cells,

155 producing a virion stock of $5 \times 10^{4} \mathrm{TCID}_{50} / \mathrm{ml}$. After intranasal inoculation with $5 \times 10^{3} \mathrm{TCID}_{50}$ of S-

156 IV-P5-Vero-P2 virion (the highest possible dose), hamsters did not lose weight or develop

157 detectable disease (Fig. S6). Collectively, the results indicate that both $\triangle O R F 3-E$ mNG virion

158 and S-IV-P5-Vero-P2 virion are highly attenuated and do not disseminate or cause disease in

159 hamsters.

160 To corroborate the hamster results, we tested $\triangle$ ORF3-E mNG virion in more susceptible

161 K18-hACE2 mice (Fig. 3G). After intranasal inoculation with $3 \times 10^{5}$ TCID $_{50}$ of $\triangle O R F 3-E$ mNG

162 virion (the highest possible dose), K18-hACE2 mice did not lose weight (Fig. 3H) or die (Fig.

$1633 \mathrm{3}$ ); in contrast, infection with $2.5 \times 10^{3} \mathrm{TCID}_{50}$ of WT SARS-CoV-2 resulted in $25 \%$ weight loss

164 and $67 \%$ lethality. To increase the stringency of the test, we inoculated K18-hACE2 mice by

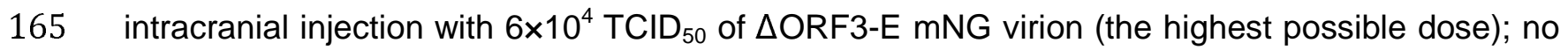

166 morbidity (Fig. 3J) or mortality (Fig. 3K) was observed. In contrast, mice inoculated by the

167 intracranial route with $500,50,5$, and $1 \mathrm{TCID}_{50}$ of WT SARS-CoV-2 developed $100 \%, 25 \%$,

$16825 \%$, and 0\% mortality, respectively (Fig. 3K). Similar to the $\triangle$ ORF3-E mNG virion, no morbidity

169 or mortality was observed after mice were inoculated by the intranasal or intracranial route with

$1702.5 \times 10^{3}$ or $5 \times 10^{2} \mathrm{TCID}_{50}$ of S-IV-P5-Vero-P2 virion, respectively (Fig. S7). Together, the results

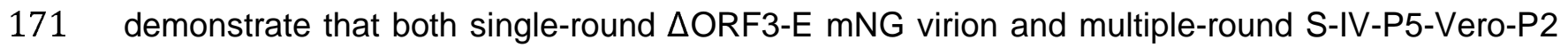

172 virion lack virulence in K18-hACE2 mice.

173 High-throughput neutralization and antiviral testing. We adapted $\triangle \mathrm{ORF}-\mathrm{E} \mathrm{mNG}$

174 virion for a high-throughput neutralization and antiviral assay. Fig. 4A outlines the assay

175 scheme in a 96-well plate format. Neutralization titers of 18 convalescent sera from COVID-19

176 patients were measured by two assays for comparison: the $\triangle \mathrm{ORF}-\mathrm{E} \mathrm{mNG}$ virion assay and the

177 gold standard plaque-reduction neutralization test (PRNT). The two assays produced 
178 comparable $50 \%$ neutralization titers $\left(\mathrm{NT}_{50}\right)$ for all specimens (Table S1 and Fig. 4B-C). In

179 addition, the $\triangle \mathrm{ORF} 3-\mathrm{E} \mathrm{mNG}$ virion assay could also be used to measure the $50 \%$ effective

180 neutralizing concentration $\left(\mathrm{EC}_{50}\right)$ for a monoclonal antibody against SARS-CoV-2 receptor-

181 binding domain (RBD; Fig. 4D). Finally, using Remdesivir as a viral polymerase inhibitor, we

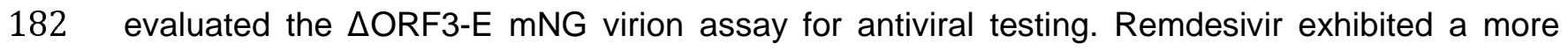

183 potent $\mathrm{EC}_{50}$ on hACE2-A549 cells $(0.27 \mu \mathrm{M}$; Fig. 4E) than that on Vero cells $(5.1 \mu \mathrm{M}$; Fig. 4F).

184 The $\mathrm{EC}_{50}$ discrepancy between the two cell types is likely due to different efficiencies in

185 converting Remdesivir to its triphosphate form, as previously reported $(3,21)$. Collectively, the

186 results demonstrate that the $\triangle \mathrm{ORF3-E}$ virion assay can be used for high-throughput

187 neutralization testing and antiviral drug discovery.

\section{DISCUSSION}

190 We generated and characterized a trans-complementation system for SARS-CoV-2. The

191 system produced a high yield of single-round infectious $\triangle$ ORF3-E virion that could be used for

192 neutralization and antiviral testing. An mNG reporter was introduced into the $\triangle O R F 3-E$ virion to

193 indicate viral replication. Depending on research needs, other reporter genes, such as luciferase

194 or GFP, could be engineered into the system. A reliable high-throughput neutralization assay is

195 important for COVID-19 vaccine evaluation and for studying the kinetics of neutralizing antibody

196 levels in post-vaccinated and naturally infected people $(4,22,23)$. Three types of cell-based

197 high-throughput neutralization assays currently are available: (i) pseudovirus assay, which

198 expresses SARS-CoV-2 S protein alone on heterologous viruses, can be performed at BSL-2

199 laboratories $(24,25)$; (ii) a reporter SARS-CoV-2 assay, which must be performed at BSL-3

200 laboratories, represents authentic viral infection $(3,5,6,26)$; (iii) bona fide fully infectious SARS-

201 CoV-2 by focus reduction neutralization test (24). The $\triangle$ ORF3-E mNG virion combines the

202 advantages of each assay type by recapitulating the authentic viral infection for a single round, 
203 thus supporting its use at BSL2 laboratories. The $\triangle$ ORF3-E mNG virion can be readily adapted

204 to investigate vaccine-elicited neutralization against newly emerged SARS-CoV-2 isolates, such

205 as the rapidly spreading United Kingdom and South African strains $(27,28)$, by swapping or

206 mutating the S gene.

The trans-complementation system can also be used for high-throughput antiviral 208 screening of large compound libraries. Infection of normal cells with $\triangle O R F 3-E m N G$ virion

209 allows for screening of inhibitors of virus entry, genome translation, and RNA replication, but not

210 virion assembly/release. In contrast, infection of Vero-ORF3-E cells with $\triangle \mathrm{ORF3-E}$ mNG virion

211 can be used to identify inhibitors of all steps of SARS-CoV-2 infection cycle, including virion

212 assembly and release; this system also allows selection for resistance to inhibitors for mode-of-

213 action studies. In addition, the single-round $\triangle$ ORF3-E virion could be developed as a safe

214 vaccine platform, as previously reported for other coronaviruses $(29,30)$.

215 Our results support that the trans-complementation system can be performed safely in

216 BSL-2 laboratories. (i) The system produced single-round infectious $\triangle O R F 3-E$ mNG virion that

217 does not infect normal cells for multiple rounds and thus cannot spread in vitro or in vivo. (ii) The

218 system did not produce WT virus, even after multiple independent selections. (iii) Although an

219 adaptive mutation in $\mathrm{M}$ protein was selected to confer multi-round infection on normal cells, the

220 replication level of such virion (i.e., S-IV-P5-Vero-P2) was barely detectable, with infectious

221 titers $>100,000$-fold lower than the WT SARS-CoV-2. The molecular mechanism of how S-IV-

222 P5-Vero-P2 could infect cells for multiple rounds without the ORF3 and E proteins remains to be

223 defined. Previous studies showed that deletion of both ORF3 and E genes was lethal for SARS-

224 CoV (31). (iv) Continuous culturing of the S-IV-P5-Vero-P2 virion on naïve Vero cells did not

225 improve viral replication. (v) When hamsters and K18-hACE2 mice were infected with the

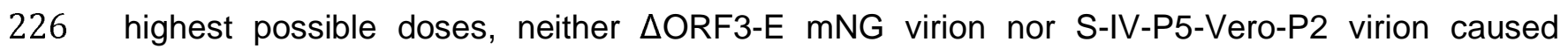

227 morbidity or mortality. Even after intracranial infection with the highest possible dose, neither 
228 virions caused detectable disease or death in the highly susceptible K18-hACE2 mice. If further

229 safety improvement is needed, more accessory ORFs could be deleted from the $\Delta$ ORF3-E

230 mNG RNA as accessory proteins are not essential for viral replication (1).

231 One limitation of our study is the use of Vero E6 cells for constructing the Vero-ORF3-E

232 cell line. When propagated on Vero E6 cells, SARS-CoV-2 could accumulate deletions at the

233 furin cleavage site in the $\mathrm{S}$ protein $(32,33)$. This cleavage deletion affects the neutralization

234 susceptibility of SARS-CoV-2 and possibly the route of entry into cells (34). Although we did not

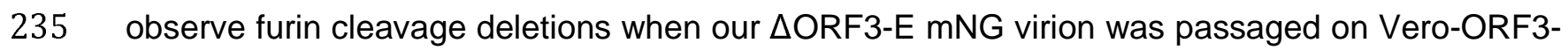

236 E cells, this possibility could be minimized or eliminated by using other cell lines, such as A549-

237 hACE2 or Vero-TMPRSS2-hACE2 cells.

238 In summary, we have developed a trans-complementation system for SARS-CoV-2 that

239 likely can be performed at BSL-2 laboratories for COVID-19 research and countermeasure

240 development. Thus, the experimental system could be used by researchers in industry,

241 academia, and government laboratories who lack access to a BSL-3 facility.

\section{METHODS}

244 Cell lines. Vero E6, Vero CCL-81, Calu-3, and HEK-293T cells were purchased from the

245 American Type Culture Collection (ATCC) and cultured in high-glucose Dulbecco's modified

246 Eagle's medium (DMEM) supplemented with $2 \mathrm{mM} \mathrm{L-glutamine,} 100 \mathrm{U} / \mathrm{ml}$ Penicillium-

247 Streptomycin (P/S), and 10\% fetal bovine serum (FBS; HyClone Laboratories, South Logan,

248 UT). Vero-ORF3-E cells were maintained in DMEM medium supplemented with 2mM L-

249 glutamine, $100 \mathrm{U} / \mathrm{ml} \mathrm{P} / \mathrm{S}, 10 \%$ FBS, $0.075 \%$ sodium bicarbonate, and $10 \mu \mathrm{g} / \mathrm{ml}$ puromycin. The

250 A549-hACE2 cells were generously provided by Shinji Makino (35) and grown in the culture 
251 medium supplemented with $10 \mu \mathrm{g} / \mathrm{mL}$ blasticidin and $10 \mathrm{mM}$ HEPES at $37{ }^{\circ} \mathrm{C}$ with $5 \% \mathrm{CO} 2$.

252 Medium and other supplements were purchased from Thermo Fisher Scientific (Waltham, MA).

253 Hamsters. Syrian golden hamsters (HsdHan:AURA strain) were purchased from Envigo.

254 Animals were housed in groups and fed standard chow diets. Hamster experiments were 255 performed as described previously (36). Briefly, $10^{5} \mathrm{TCID}_{50}$ of WT SARS-CoV-2, $6 \times 10^{5} \mathrm{TCID}_{50}$

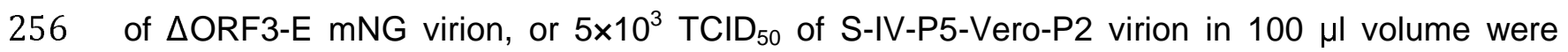
257 inoculated into four- to five-week-old male Syrian golden hamsters via the intranasal route. The 258 S-IV-P5-Vero-P2 virion stock was prepared by two rounds of culturing of S-IV-P5 virion (Fig.

259 S4A) on Vero E6 cells (to remove single-round infectious virion), followed by propagation on

260 Vero-ORF3-E cells. Fourteen hamsters were used in SARS-CoV-2- and $\triangle$ ORF3-E mNG virion-

261 infected groups and 5 hamsters were used in S-IV-P5-Vero-P2 virion-infected group. From day

2621 to 14 post-infection, hamsters were observed daily for weight change and signs of illness. Five

263 hamsters in WT SARS-CoV-2-, $\triangle$ ORF3-E mNG virions-, or mock-infected group were sacrificed

264 on day 2 post-infection for lung and trachea collections. Nasal washes and oral swabs of the

265 rest 9 hamsters per group were collected on days 2, 4, and 7 post-infection.

266 Mice. Animal studies were carried out in accordance with the recommendations in the

267 Guide for the Care and Use of Laboratory Animals of the National Institutes of Health. The 268 protocols were approved by the Institutional Animal Care and Use Committee at the Washington 269 University School of Medicine (assurance number A3381-01). Heterozygous K18-hACE 270 c57BL/6J mice (strain: 2B6.Cg-Tg(K18-ACE2)2Prlmn/J) were obtained from the Jackson

271 Laboratory. Animals were randomized upon arrival at Washington University and housed in

272 groups of $<5$ per cage in rooms maintained between $68-74^{\circ} \mathrm{F}$ with $30-60 \%$ humidity and 273 day/night cycles of $12 \mathrm{~h}$ intervals (on 6AM-6PM). Mice were fed standard chow diets. Mice 7-9

274 weeks of age and of both sexes were used for this study. Intranasal virus inoculations (50

$275 \mathrm{uL} /$ mouse) were performed under sedation with ketamine hydrochloride and xylazine while 
276 intracranial virus inoculations (10 $\mu \mathrm{L} /$ mouse) were performed under sedation with isoflurane; all

277 efforts were made to minimize animal suffering.

278 Plasmid construction. Seven previously reported subclone plasmids for the assembly 279 of the entire genome of SARS-CoV-2 were used in this study, including pUC57-F1, pCC1-F2, 280 pCC1-F3, pUC57-F4, pUC57-F5, pUC57-F6, and pCC1-F7-mNG (2, 26). For the convenience 281 of deleting ORF3-E gene, we constructed F5, F6, and F7-mNG fragments into one plasmid. F5,

282 F6, and F7-mNG fragments were amplified from corresponding subclones via PCR with primer 283 pairs pcov-F56-F1/pncov-R5, pncov-F6/pncov-R6, and pncov-F7/pncov-R8, respectively (Table 284 S2). All PCR products were cloned together into a $\mathrm{pCC} 1$ vector through Notl and Clal restriction 285 sites using the standard restriction digestion-ligation cloning, resulting in subclone pCC1-F567$286 \mathrm{mNG}$.

To introduce ORF3-E deletion and mutant Transcription Regulatory Sequence (TRS) 288 into pCC1-F567-mNG, seven fragments were amplified with primer pairs cov-21115-F/TRS2-S289 R, TRS2-S-F/S-TRS2-M-R，TRS2-M-F/M-TRS2-R， M-TRS2-F/ORF6-TRS2-mNG-R， ORF6290 TRS2-mNG-F/ORF7-TRS2-ORF8-R, ORF7-TRS2-ORF8-F/ORF8-TRS2-N-R, and TRS2-N-

291 F/cov-28501-R. The seven PCR products were assembled into the pCC1-F567-mNG plasmid 292 that were pre-linearized with Nhel and Xhol by using the NEBuilder® HiFi DNA Assembly kit 293 (NEB) according to the manufacturer's instruction, resulting in subclone pCC1-F567-mNG$294 \Delta$ ORF3-E. Mutation T130N in M protein was engineered into pCC1-F567-mNG- $\triangle$ ORF3-E with 295 primers M-T130N-F/M-T130N-R via overlap PCR. Mutant TRS was engineered into pCC1-F1 296 with primers 5 $\square$ UTR-TRS2-F and 5 $\square$ UTR-TRS2-R via overlap PCR.

297 For making the Vero-ORF3-E cell lines, codon-optimized SARS-CoV-2 ORF3 and E 298 genes were synthesized by GenScript Biotech (Piscataway, NJ). An mCherry reporter Zika virus 299 cDNA plasmid (37) was used as a template to amplify the mCherry-F2A gene. For constructing 300 a lentiviral plasmid expressing ORF3 and E protein of SARS-CoV-2, DNA fragments encoding 301 mCherry-F2A, SARS-CoV-2 E, EMCV IRES, and SARS-CoV-2 ORF3 were amplified with 
302 primers EcoR1-mCherry-F/F2A-optE-R, F2A-optE-F/EcoR1-Cov-optE-R, EcoR1-IRES-F/EMCV-

303 IRES-R, and IRES-optORF3-F/BamH1-Cov-optORF3-R, respectively. The PCR products then

304 were inserted into a Tet-on inducible lentiviral vector pLVX (Takara, Mountain View, CA)

305 through EcoRI and BamHI restriction sites, resulting in plasmid pLVX-ORF3-E.

306 Selection of Vero-ORF3-E cell line. For packaging the lentivirus, the pLVX-ORF3-E

307 plasmid was transfected into HEK-293T cells using the Lenti-X Packaging Single Shots kit

308 (Takara). Lentiviral supernatants were harvested at $72 \mathrm{~h}$ post-transfection and filtered through a

$3090.22 \mu \mathrm{M}$ membrane (Millipore, Burlington, MA). One day before transduction, Vero E6 cells were

310 seeded in a 6 -well plate $\left(4 \times 10^{5}\right.$ per well) with DMEM containing $10 \%$ FBS. After $12-18 \mathrm{~h}$, cells

311 were transduced with $2 \mathrm{ml}$ lentivirus for $24 \mathrm{~h}$ in the presence of $12 \mu \mathrm{g} / \mathrm{ml}$ of polybrene (Sigma-

312 Aldrich, St. Louis, MO). At $24 \mathrm{~h}$ post-transduction, cells from a single well were split into four 10

$313 \mathrm{~cm}$ dishes and cultured in medium supplemented with $25 \mu \mathrm{g} / \mathrm{ml}$ of puromycin. The culture

314 medium containing puromycin was refreshed every 2 days. After 2-3 weeks of selection, visible

315 puromycin-resistant cell colonies were formed. Several colonies were transferred into 24-well

316 plates. When confluent, cells were treated with trypsin and seeded in 6-well plates for further

317 expansion. The resulting cells were defined as Vero-ORF3-E P0 cells. For cell line verification,

318 total cellular mRNA was isolated and subject to RT-PCR with primers EcoR1-mCherry-F and

319 BamH1-Cov-optORF3-R (Table S2), followed by cDNA sequencing of the ORF3-E genes.

$\triangle$ ORF3-E mNG cDNA assembly and in vitro RNA transcription. Full-length genome

321 assembly and RNA transcription were performed as described previously with minor

322 modifications (2). Briefly, individual subclones containing fragments of the $\triangle O R F 3-E$ mNG viral

323 genome were digested with appropriated restriction endonucleases and resolved in a $0.8 \%$

324 agarose gel. Specifically, the plasmids containing F1, F2, F3, or F4 fragments were digested

325 with Bsal enzyme, and the plasmid containing F567-mNG- $\triangle$ ORF3-E fragment was digested

326 with Espl enzyme. All fragments were recovered using the QIAquick Gel Extraction Kit

327 (QIAGEN, Hilden, Germany), and total of $5 \mu \mathrm{g}$ of the five fragments was ligated in an equal 
328 molar ratio by T4 DNA ligase (New England Biolabs, Ipswich, MA) at $4^{\circ} \mathrm{C}$ overnight. Afterward,

329 the assembled full-length genomic cDNA was purified by phenol-chloroform extraction and 330 isopropanol precipitation. $\triangle$ ORF3-E mNG RNA transcripts were generated using the T7 331 mMessage mMachine kit (Ambion, Austin, TX). To synthesize the $\mathrm{N}$ gene RNA transcript of 332 SARS-CoV-2, the $\mathrm{N}$ gene was PCR-amplified by primers CoV-T7-N-F and polyT-N-R (Table 333 S2) from a plasmid containing the F7 fragment (2); the PCR product was then used for in vitro 334 transcription using the T7 mMessage mMachine kit (Ambion).

$335 \Delta$ ORF3-E $\mathbf{m N G}$ virion production and quantification. Vero-ORF3-E cells were 336 seeded in a T175 flask and grown in DMEM medium with $100 \mathrm{ng} / \mathrm{ml}$ of doxycycline. On the next 337 day, $40 \mu \mathrm{g}$ of $\triangle$ ORF3-E mNG RNA and $20 \mu \mathrm{g}$ of $\mathrm{N}$-gene RNA were electroporated into $8 \times 10^{6}$ 338 Vero-ORF3-E cells using the Gene Pulser XCell electroporation system (Bio-Rad, Hercules, $339 \mathrm{CA})$ at a setting of $270 \mathrm{~V}$ and $950 \mu \mathrm{F}$ with a single pulse. The electroporated cells were then 340 seeded in a T75 flask and cultured in the medium supplemented with doxycycline (Sigma341 Aldrich) at $37^{\circ} \mathrm{C}$ for $3-4$ days. Virion infectivity was quantified by measuring the $\operatorname{TCID}_{50}$ using an 342 end-point dilution assay as previously reported (38). Briefly, Vero-ORF3-E cells were plated on 343 96-well plates $\left(1.5 \times 10^{4}\right.$ per well) one day prior to infection. The cells were cultured in medium 344 with doxycycline as described above. $\triangle$ ORF3-E mNG virions were serially diluted in DMEM 345 medium supplemented with $2 \%$ FBS, with 6 replicates per concentration. Cells were infected 346 with $100 \mu \mathrm{l}$ of diluted virions and incubated at $37^{\circ} \mathrm{C}$ for $2-3$ days. The $\mathrm{mNG}$ signals were 347 counted under a fluorescence microscope (Nikon, Tokyo, Japan). TCID 50 was calculated using 348 the Reed \& Muench method (39).

349 To assess viral RNA levels, a quantitative RT-PCR assay was conducted using an iTaq 350 Universal SYBR Green one-step kit (Bio-Rad) on a QuantStudio 7 Flex Real-Time PCR 351 Systems (Thermo fisher) by following the manufacturers' protocols. Primers CoV19-N2-F and

352 CoV19-N2-R (Table S2) targeting the $\mathrm{N}$ gene were used. Absolute RNA copies were 
353 determined by standard curve method using in vitro transcribed RNA containing genomic 354 nucleotide positions 26,044 to 29,883 of the SARS-CoV-2 genome.

355 RNA extraction, RT-PCR, and cDNA sequencing. Supernatants of infected cells were 356 collected and centrifuged at $1,000 \mathrm{~g}$ for $10 \mathrm{~min}$ to remove cell debris. Clarified culture fluids (250

$357 \mu \mathrm{l})$ were mixed thoroughly with $1 \mathrm{ml}$ of TRIzol LS reagent (Thermo Fisher Scientific).

358 Extracellular RNA was extracted per manufacture's instruction and resuspended in $20 \mu \mathrm{l}$ of

359 nuclease-free water. RT-PCR was performed using the SuperScript@ IV One-Step RT-PCR kit

360 (Thermo Fisher Scientific). Nine cDNA fragments ( $\mathrm{FF} 1$ to $\mathrm{gF9}$ ) covering the whole viral genome

361 were generated with specific primers according to the protocol described previously (2).

362 Afterward, cDNA fragments were separated in a 0.8\% agarose gel, purified using QIAquick Gel

363 Extraction Kit (QIAGEN), and subjected to Sanger sequencing.

$364 \Delta$ ORF3-E mNG virion neutralization assay. The research protocol for use of human serum specimens was approved by the University of Texas Medical Branch (UTMB) Institutional

366 Review Board (IRB protocol number 20-0070). All human serum specimens were obtained at 367 the UTMB with patient information de-identified. For neutralization testing, Vero CCL-81 cells $368\left(1.2 \times 10^{4}\right)$ in $50 \mu$ l of DMEM containing $2 \%$ FBS and $100 \mathrm{U} / \mathrm{ml} \mathrm{P/S}$ were seeded in each well of 369 black $\mu$ CLEAR flat-bottom 96-well plate (Greiner Bio-one ${ }^{\mathrm{TM}}$, Kremsmünster, Austria). At 16 h 370 post-seeding, $30 \mu \mathrm{L}$ of 2 -fold serial diluted human sera were mixed with $30 \mu \mathrm{L}$ of $\Delta \mathrm{ORF}-\mathrm{E}$ $371 \mathrm{mNG}$ virion (MOI of 5 ) and incubated at $37^{\circ} \mathrm{C}$ for $1 \mathrm{~h}$. Afterward, $50 \mu \mathrm{L}$ of virus-sera complexes 372 were transferred to each well of the 96 -well plate. After incubating the infected cells at $37^{\circ} \mathrm{C}$ for $37320 \mathrm{~h}, 25 \mu \mathrm{l}$ of Hoechst 33342 Solution (400-fold diluted in Hank's Balanced Salt Solution; 374 Thermo Fisher Scientific) were added to each well to stain the cell nucleus. The plate was 375 sealed with Breath-Easy sealing membrane (Diversified Biotech, Dedham, MA), incubated at $37637^{\circ} \mathrm{C}$ for $20 \mathrm{~min}$, and quantified for mNG-positive cells using the Celllnsight $\mathrm{CX} 5$ High-Content 377 Screening Platform (Thermo Fisher Scientific). Infection rates were determined by dividing the 378 mNG-positive cell number to the total cell number. Relative infection rates were obtained by 
379 normalizing the infection rates of serum-treated groups to those of non-serum-treated controls.

380 The curves of the relative infection rates versus the serum dilutions (log10 values) were plotted 381 using Prism 9 (GraphPad, San Diego, CA). A nonlinear regression method was used to 382 determine the dilution fold that neutralized $50 \%$ of $\mathrm{mNG}$ fluorescence $\left(\mathrm{NT}_{50}\right)$. Each serum was 383 tested in duplicates.

$384 \Delta$ ORF3-E mNG virion for mAb and antiviral testing. Vero CCL-81 cells $\left(1.2 \times 10^{4}\right)$ or 385 A549-hACE2 cells in $50 \mu$ of culture medium containing $2 \%$ FBS were seeded in each well of 386 black $\mu$ CLEAR flat-bottom 96-well plate. At $16 \mathrm{~h}$ post-seeding, 2- or 3-fold serial diluted human 387 mAb14 (40) or Remdesivir were mixed with $\triangle$ ORF3-E mNG virion (MOI of 1 ). Fifty microliters of 388 mixtures were transferred to each well of the 96-well plate. After incubating the infected cells at $38937^{\circ} \mathrm{C}$ for $20 \mathrm{~h}, 25 \mu$ of Hoechst 33342 Solution (400-fold diluted in Hank's Balanced Salt

390 Solution) were added to each well to stain the cell nucleus. The plate was sealed with Breath-

391 Easy sealing membrane, incubated at $37^{\circ} \mathrm{C}$ for $20 \mathrm{~min}$. mNG-positive cells were quantified and

392 infection rates were calculated as described above. Relative infection rates were obtained by 393 normalizing the infection rates of treated groups to those of non-treated controls. For

394 Remdesivir, $0.1 \%$ of DMSO-treated groups were used as controls. A nonlinear regression 395 method was used to determine the concentration that inhibited $50 \%$ of mNG fluorescence $396\left(\mathrm{EC}_{50}\right)$. Experiments were performed in triplicates or quadruplicates.

397 Biosafety. All aspects of this study were approved by the Institutional Biosafety 398 Committee of the University of Texas Medical Branch at Galveston before the initiation of this 399 study. Experiments with SARS-CoV-2, trans-complementation, and $\triangle$ ORF3-E mNG virion were 400 performed in a BSL-3 laboratory by personnel equipped with powered air-purifying respirators.

401 Transmission Electron Microscopy. Supernatants of infected cells were centrifuged

402 for $10 \mathrm{~min}$ at $3,000 \mathrm{~g}$ to remove cellular debris. Nickel grids were incubated with clarified 403 supernatants for 10 min followed by glutaraldehyde fixation and $2 \%$ uranyl acetate staining. 
404 Micrographs were taken using a JEM 1400 (JEOL USA Inc.). Multiple randomly selected fields 405 were imaged.

406 Bioinformatics analysis. Fluorescence images were processed using ImageJ (41).

407 Virus sequences were download from the NCBI database and aligned using Geneious software.

408 DNA gel images were analyzed using Image Lab software. Statistical graphs or charts were 409 created using the GraphPad Prism 9 software. Figures were created and assembled using 410 BioRender and Adobe illustration (San Jose, CA).

411 Statistical analysis. A linear regression model in the software Prism 9 (GraphPad) was 412 used to calculate the $\mathrm{NT}_{50}$ and $\mathrm{EC}_{50}$ values from the $\triangle \mathrm{ORF}-\mathrm{E}$ virion assay. Pearson 413 correlation coefficient and two-tailed p-value are calculated using the default settings in the 414 software Prism 9. An unpaired T-test (for two-groups comparison) and ANOVA test (for multi415 group comparison) were used in statistical analysis $\left(^{*}, \mathrm{P}<0.05\right.$, significant; ${ }^{*}$, $\mathrm{P}<0.01$, very 416 significant; ${ }^{* * *}, \mathrm{P}<0.001$, highly significant; ${ }^{* * *}, \mathrm{P}<0.0001$, extremely significant; ns, $\mathrm{P}>0.05$, not 417 significant).

\section{DATA AVAILABILITY}

The results presented in the study are available upon request from the corresponding

421 authors. The mNG reporter SARS-CoV-2 has been deposited to the World Reference Center for

422 Emerging Viruses and Arboviruses (https://www.utmb.edu/wrceva) at UTMB for distribution.

423

424

425

426

427

428

429

430

431

432

433

\section{REFERENCES}

1. B. Hu, H. Guo, P. Zhou, Z. L. Shi, Characteristics of SARS-CoV-2 and COVID-19. Nat Rev Microbiol, (2020).

2. X. Xie et al., An Infectious cDNA Clone of SARS-CoV-2. Cell Host Microbe 27, 841-848 e843 (2020).

3. X. Xie et al., A nanoluciferase SARS-CoV-2 for rapid neutralization testing and screening of anti-infective drugs for COVID-19. Nat Commun 11, 5214 (2020).

4. M. J. Mulligan et al., Phase I/II study of COVID-19 RNA vaccine BNT162b1 in adults. Nature 586, 589-593 (2020). 
$434 \quad 5 . \quad$ Y. J. Hou et al., SARS-CoV-2 Reverse Genetics Reveals a Variable Infection Gradient in

435

436

437

438 the Respiratory Tract. Cell 182, 1-18 (2020).

6. T. Thi Nhu Thao et al., Rapid reconstruction of SARS-CoV-2 using a synthetic genomics platform. Nature 582, 561-565 (2020).

7. H. Yao et al., Molecular Architecture of the SARS-CoV-2 Virus. Cell 183, 730-738 e713 (2020).

8. F. Ge, Y. Luo, P. X. Liew, E. Hung, Derivation of a novel SARS-coronavirus replicon cell line and its application for anti-SARS drug screening. Virology 360, 150-158 (2007).

9. A. A. Khromykh, E. G. Westaway, Subgenomic replicons of the flavivirus Kunjin: construction and applications. J. Virol. 71, 1497-1505 (1997).

10. V. Lohmann et al., Replication of subgenomic hepatitis $\mathrm{C}$ virus RNAs in a hepatoma cell line. Science 285, 110-113 (1999).

11. L. Lo, M. Tilgner, P.-Y. Shi, A potential high-throughput assay for screening inhibitors of West Nile virus replication. J. Virol. 77, 12901-12906 (2003).

12. T. Hertzig et al., Rapid identification of coronavirus replicase inhibitors using a selectable replicon RNA. J Gen Virol 85, 1717-1725 (2004).

13. H. Xia et al., Evasion of Type I Interferon by SARS-CoV-2. Cell Rep 33, 108234 (2020).

14. R. L. Graham, D. J. Deming, M. E. Deming, B. L. Yount, R. S. Baric, Evaluation of a recombination-resistant coronavirus as a broadly applicable, rapidly implementable vaccine platform. Commun Biol 1, 179 (2018).

454

455

15. B. Yount, R. S. Roberts, L. Lindesmith, R. S. Baric, Rewiring the severe acute respiratory syndrome coronavirus (SARS-CoV) transcription circuit: engineering a recombination-resistant genome. Proc Natl Acad Sci U S A 103, 12546-12551 (2006).

16. R. Mahtarin et al., Structure and dynamics of membrane protein in SARS-CoV-2. J Biomol Struct Dyn, 1-14 (2020).

17. S. Thomas, The Structure of the Membrane Protein of SARS-CoV-2 Resembles the Sugar Transporter SemiSWEET. Pathog Immun 5, 342-363 (2020).

18. E. S. Winkler et al., SARS-CoV-2 infection of human ACE2-transgenic mice causes severe lung inflammation and impaired function. Nat Immunol 21, 1327-1335 (2020).

19. J. F. Chan et al., Simulation of the clinical and pathological manifestations of Coronavirus Disease 2019 (COVID-19) in golden Syrian hamster model: implications for disease pathogenesis and transmissibility. Clin Infect Dis, (2020).

20. P. B. McCray, Jr. et al., Lethal infection of K18-hACE2 mice infected with severe acute respiratory syndrome coronavirus. J Virol 81, 813-821 (2007).

21. A. J. Pruijssers et al., Remdesivir Inhibits SARS-CoV-2 in Human Lung Cells and Chimeric SARS-CoV Expressing the SARS-CoV-2 RNA Polymerase in Mice. Cell Rep 32, 107940 (2020).

22. E. E. Walsh et al., Safety and Immunogenicity of Two RNA-Based Covid-19 Vaccine Candidates. N Engl J Med, (2020).

23. A. T. Widge et al., Durability of Responses after SARS-CoV-2 mRNA-1273 Vaccination. N Engl J Med, (2020).

24. J. B. Case et al., Neutralizing Antibody and Soluble ACE2 Inhibition of a ReplicationCompetent VSV-SARS-CoV-2 and a Clinical Isolate of SARS-CoV-2. Cell Host Microbe 28, 475-485 e475 (2020).

25. C. Zeng et al., Neutralizing antibody against SARS-CoV-2 spike in COVID-19 patients, health care workers, and convalescent plasma donors. JCI Insight 5, (2020).

26. A. E. Muruato et al., A high-throughput neutralizing antibody assay for COVID-19 diagnosis and vaccine evaluation. Nat Commun 11, 4059 (2020).

27. K. Kupferschmidt, Fast-spreading U.K. virus variant raises alarms. Science 371, 9-10 (2021). 
28. Xie, X. et al., Neutralization of N501Y mutant SARS-CoV-2 by BNT162b2 vaccineelicited sera. BioRxiv, doi: https://doi.org/10.1101/2021.1101.1107.425740 (2021).

29. F. Almazan et al., Engineering a replication-competent, propagation-defective Middle East respiratory syndrome coronavirus as a vaccine candidate. mBio 4, e00650-00613 (2013).

30. J. Ortego, D. Escors, H. Laude, L. Enjuanes, Generation of a replication-competent, propagation-deficient virus vector based on the transmissible gastroenteritis coronavirus genome. J Virol 76, 11518-11529 (2002).

31. C. Castano-Rodriguez et al., Role of Severe Acute Respiratory Syndrome Coronavirus Viroporins E, 3a, and 8a in Replication and Pathogenesis. mBio 9, (2018).

32. S. Y. Lau et al., Attenuated SARS-CoV-2 variants with deletions at the S1/S2 junction. Emerg Microbes Infect 9, 837-842 (2020).

33. W. B. Klimstra et al., SARS-CoV-2 growth, furin-cleavage-site adaptation and neutralization using serum from acutely infected hospitalized COVID-19 patients. J Gen Virol 101, 1156-1169 (2020).

34. B. A. Johnson et al., Furin Cleavage Site Is Key to SARS-CoV-2 Pathogenesis. bioRxiv, (2020).

35. E. C. Mossel et al., Exogenous ACE2 expression allows refractory cell lines to support severe acute respiratory syndrome coronavirus replication. J Virol 79, 3846-3850 (2005).

504

505

506

507

508

509

510

511

512

513

514

515

36. J. A. Plante et al., Spike mutation D614G alters SARS-CoV-2 fitness. Nature, (2020).

37. C. Shan et al., A live-attenuated Zika virus vaccine candidate induces sterilizing immunity in mouse models. Nat Med, (2017).

38. B. D. Lindenbach, Measuring HCV infectivity produced in cell culture and in vivo. Methods Mol Biol 510, 329-336 (2009).

39. L. J. Reed, H. Muench, A simple method of estimating fifty percent endpoints. The American Journal of Hygiene 27, 493-497 (1938).

40. Z. Ku et al., Molecular determinants and mechanism for antibody cocktail preventing SARS-CoV-2 escape. Nature Communications, https://doi.org/10.1038/s41467-4102020789-41467 (2021).

41. C. A. Schneider, W. S. Rasband, K. W. Eliceiri, NIH Image to ImageJ: 25 years of image analysis. Nat Methods 9, 671-675 (2012).

\section{ACKNOWLEDGEMENTS}

We thank John Bilello from Gilead for providing Remdesivir and Zhiqiang An from the

518 University of Texas Health Science at Houston for providing mAb14. P.-Y.S. was supported by

519 NIH grants Al142759, Al134907, Al145617, and UL1TR001439; awards from the Sealy \& Smith

520 Foundation, Kleberg Foundation, John S. Dunn Foundation, Amon G. Carter Foundation, Gilson

521 Longenbaugh Foundation, and Summerfield Robert Foundation; and fund in sponsored

522 research agreement from $Q^{2}$ Solutions. M.S.D. was supported by R01 Al157155. V.D.M. was

523 supported by NIH grants U19Al100625, R00AG049092, R24Al120942, and a STARs Award

524 from the University of Texas System. S.C.W. was supported by NIH grant R24 Al120942. J.L. is 
525 supported by the postdoctoral fellowship from the McLaughlin Fellowship Endowment at

526 UTMB. P.R. and X.X. were partially supported by the Sealy \& Smith Foundation.

528 AUTHOR CONTRIBUTIONS

529 X.Z., V.D.M., X.X., and P.-Y.S conceived the study. X.Z., Y.L., J.L., A.L.B., K.S.P.,

530 J.A.P., J.Z., H.X., N.B., P.R., and X.X. performed the experiments. X.Z., Y.L., A.L.B., P.A., P.R.,

531 V.D.M., M.S.D., S.W., X.X., and P.-Y.S. analyzed the results. P.R. prepared the serum

532 specimens. X.Z., Y.L., A.L.B., V.D.M., M.S.D., S.W., X.X., and P.-Y.S wrote the manuscript.

533

534 COMPETING INTERESTS

535 X.Z., X.X., and P.-Y.S. have filed a patent on the trans-complementation system of

536 SARS-CoV-2. M.S.D. is a consultant for Inbios, Vir Biotechnology, NGM Biopharmaceuticals,

537 and Carnival Corporation, and on the Scientific Advisory Boards of Moderna and Immunome.

538 The Diamond laboratory has received unrelated funding support in sponsored research

539 agreements from Moderna, Vir Biotechnology, and Emergent BioSolutions. 
Figure 1. Generation of single-round infectious $\triangle$ ORF3-E $\mathrm{mNG}$ virion (A) A transcomplementation system for SARS-CoV-2. Vero-ORF3-E cells are electroporated with $\triangle$ ORF3E mNG RNA. Trans-complementation produces $\triangle$ ORF3-E mNG virion (left panel) which can infect naïve Vero E6 cells for only single round (right panel). (B) $\triangle$ ORF3-E mNG virion genome. Both the full-length mNG SARS-CoV-2 genome (top panel) and the $\triangle O R F 3-E \mathrm{mNG}$ virion genome (bottom panel) are shown. The genomic fragment 8 (gF8) of RT-PCR analysis are indicated above both genomes. The ORF3-E deletion junction is indicated. The WT and mutant Transcription Regulatory Sequences (TRS) are also depicted. (C) ORF3-E RNA expression in Vero-ORF3-E cells. Doxycycline (Dox) was used to induce the expression of ORF3-E RNA. RTPCR analyses were performed on Vero-ORF3-E cells with or without doxycycline induction as well as on naïve Vero E6 cells. (D) Induction of mCherry expression in Vero-ORF3-E cells. Passage 1 (P1) and 20 (P20) of Vero-ORF3-E cells were induced by doxycycline to express mCherry fluorescence. Scale bar, $100 \mu \mathrm{m}$. (E) Production of $\triangle$ ORF3-E mNG virion after electroporation. After electroporating $\triangle \mathrm{ORF3-E} \mathrm{mNG} R N A$ into Vero-ORF3-E cells (with doxycycline), infectious titers of $\triangle O R F 3-E \mathrm{mNG}$ virion were measured from culture medium. Three sets of repeated experiments are presented with bars representing standard deviations. (F) Negative-staining electron microscopic image of $\triangle O R F 3-E \mathrm{mNG}$ virion. Scale bar, $50 \mathrm{~nm}$. (G) Analysis of $\triangle O R F 3-E$ mNG virion infection. Vero E6 or Vero-ORF3-E cells were incubated with WT mNG SARS-CoV-2 or $\triangle$ ORF3-E mNG virion for $2 \mathrm{~h}$. The cells were washed three times with PBS to remove residual input virus. At $48 \mathrm{~h}$ post-infection, the supernatants of the infected cells were transferred to fresh Vero E6 or Vero-ORF3-E cells for a second round of infection. The mNG signals from both rounds of infected cells are presented. Scale bar, $100 \mu \mathrm{m}$. (H) RTPCR analysis. Extracellular RNA from the second-round infection from $(\mathbf{G})$ was harvested at 48 h post-infection. Fragment 8 of the viral genome, depicted in (B), was amplified by RT-PCR to confirm the ORF3-E deletion and $\mathrm{mNG}$ retention.

Figure 2. Adaptive mutations to improve the yield of $\triangle$ ORF3-E $\mathrm{mNG}$ virion production. (A) Viral replication kinetics on Vero-ORF3-E cells. Adaptive mutations (D) were selected by continuously passaging the $\triangle O R F 3-E$ virion on Vero-ORF3-E cells for 10 rounds. For comparing the replication kinetics of the passaged viruses, Vero-ORF3-E cells were infected with the P1 or P10 $\triangle$ ORF3-E virion, $\triangle$ ORF3-E virion containing an S mutation in (D) $[\triangle O R F 3-E$ virion mut-S], or $\triangle$ ORF3-E virion containing all adaptive mutations in nsp1, nsp4, and $S$ in (D) [ $\triangle$ ORF3-E virion mut-All] at an MOI of 0.15. WT mNG SARS-CoV-2 was included as a control. Viral titers in culture supernatants are presented. ANOVA with multiple comparison correction test were performed with ${ }^{*}, \mathrm{P}<0.05$; ${ }^{* *}, \mathrm{P}<0.01$. (B) mNG-positive cells at 24 and $48 \mathrm{~h}$ postinfection from (A). Scale bar, $100 \mu \mathrm{m}$. (C) RT-PCR analysis for single-round infection. For confirming the P10 $\triangle$ ORF3-E virion remains infectious for only a single round on Vero cells, Vero E6 or Vero-ORF3-E cells were infected with WT mNG SARS-CoV-2 or P10 $\triangle$ ORF3-E $\mathrm{mNG}$ virion for two rounds as described in Fig. 1G. Viral RNAs were extracted from the secondround culture fluids and analyzed by RT-PCR. The RT-PCR product, fragment 8 ( $\mathrm{gF} 8$ ), is indicated in Fig. 1B. (D) Adaptive mutations. Three mutations were identified from whole genome sequencing of P10 $\triangle$ ORF3-E mNG virion. No mutation was found in the P1 $\triangle O R F 3-E$ $\mathrm{mNG}$ virion.

Figure 3. Safety characterization of $\triangle$ ORF3-E mNG virion in animal models. (A) Hamster experimental schedule. Four- to five-week-old male Syrian golden hamsters were intranasally (I.N.) inoculated with $10^{5} \mathrm{TCID}_{50}$ of WT SARS-CoV-2, $6 \times 10^{5} \mathrm{TCID}_{50}$ of $\triangle$ ORF3-E mNG virion, or PBS mock control. Hamsters were monitored for weight loss, disease, and viral RNA level. (B) Hamster weight change $(n=9)$. (C) Hamster disease $(n=9)$. (D) Hamster nasal wash viral RNA 
591

592

593

594

595

596

597

598

599

600

601

602

603

604

605

606

607

608

609

610

611

612

613

614

615

616

617

618

619

620

621

622

623

624

625

626

627

628

629

630

631

632

633

634

635

636

637

638

639

640

level $(n=9)$. (E) Hamster oral swab viral RNA level $(n=9)$. (F) Viral RNA loads in hamster trachea and lung at day 2 post-infection $(n=5)$. Limit of detection (L.O.D.) was defined as the RNA copies detected from mock-infected hamster samples. The weight loss data are shown as mean \pm standard deviation and statistically analyzed using two-way ANOVA Turkey's multiple comparison. The genomic RNA levels are presented as mean \pm standard error of the mean and analyzed by Mann-Whitney test. *, $\mathrm{P}<0.05$; ${ }^{* *}, \mathrm{P}<0.01$; ${ }^{* *}, \mathrm{P}<0.001$; ${ }^{* * *}, \mathrm{P}<0.0001$. (G) Mouse experimental schedule. Seven- to nine-week-old K18-hACE2 mice were inoculated with WT SARS-CoV-2 or $\triangle$ ORF3-E mNG virion via the intranasal (I.N.) or intracranial (I.C.) route. Mouse weight loss after I.N. (H) or I.C. (J) infection. Body weights were normalized to the initial weight. The means for each group [I.N: WT SARS-CoV-2 $(n=9), \triangle$ ORF3-E mNG virion $(n=4)$, and mock $(n=4)$; I.C: WT SARS-CoV-2 $500 \operatorname{TCID}_{50}(n=4), 50 \operatorname{TCID}_{50}(n=5), 5 \operatorname{TCID}_{50}(n=5)$, and $1 \operatorname{TCID}_{50}$ $(n=5)$ and $6 \times 10^{4} \operatorname{TCID}_{50} \triangle$ ORF3-E mNG virus $\left.(n=4)\right]$ are indicated, with error bars indicating the standard deviation. A mixed-model ANOVA using Dunnett's test for multiple comparisons was used to evaluate the statistical significance among groups: ${ }^{*}, \mathrm{P}<0.05 ;{ }^{* *}, \mathrm{P}<0.01 ;{ }^{* * *}, \mathrm{P}<0.001$; $* * * *, P<0.0001$. Mouse survival after I.N. (I) and I.C. (K) inoculation was analyzed using the Gehan-Breslow-Wilcoxon test. Using groups with 100\% survival as a comparator, a Bonferroni correction was applied manually to adjust the threshold for significance (indicated by ${ }^{*}$ ).

Figure 4. $\triangle$ ORF3-E $\mathrm{mNG}$ virion-based high-throughput neutralization and antiviral testing. (A) Assay scheme in a 96-well format. (B) Correlation analysis of $\mathrm{NT}_{50}$ values between the $\triangle$ ORF3-E mNG virion assay and plaque-reduction neutralization test (PRNT). The Pearson correlation efficiency $R^{2}$ and $P$ values (two-tailed) are indicated. (C) Neutralization curves. Representative curves are presented for one negative and three positive sera. The means and standard deviations from two independent experiments are shown. (D) $E_{50}$ of human mAb14 against $\triangle$ ORF3-E mNG virion infecting Vero CCL81 cells. The mean \pm standard deviations from four independent experiments are indicated. (E) $E_{50}$ of Remdesivir against $\triangle O R F 3-E m N G$ virion infecting A549-hACE2 cells. (F) $E_{50}$ of Remdesivir against $\triangle O R F 3-E \mathrm{mNG}$ virion on Vero CCL81 cells. For $(\mathbf{E})$ and $(\mathbf{F})$, the mean \pm standard deviations from three independent experiments are indicated. The four-parameter dose-response curve was fitted using the nonlinear regression method.

\section{SUPPLEMENTAL FIGURE LEGENDS}

Figure S1. Construction of Vero-ORF3-E cell lines. (A) Construction of a lentiviral transfer plasmid encoding mCherry, ORF3, and $E$ protein. The sequence of FMDV $2 A$ and its translational break position is indicated by an arrow. (B) Merged mCherry (red) and nuclei (blue) images of 3 selected clones of Vero-ORF3-E cell lines. Nuclei were stained with Hoechst 33342. Doxycycline induction is indicated. (C) mCherry expression in doxycycline-induced cells. mCherry-positive cells were quantified using a plate reader. The percentages of mCherry positive cells are presented. The results are presented as means \pm standard deviations from six replicates, and more than $10^{5}$ cells were counted for each clone. Clone 1 was used in the rest of this study.

Figure S2. Single-round infection of $\triangle$ ORF3-E mNG virion. (A) Calu-3 and A549-hACE2 cells (MOI of 1 and 10 for $\mathrm{mNG}$ SARS-CoV-2 and $\triangle$ ORF3-E mNG virion, respectively; viral titers determined on Vero-ORF3-E cells) were infected with mNG SARS-CoV-2 or $\triangle$ ORF3-E mNG virion for $2 \mathrm{~h}$, after which the cells were washed and cultured in fresh medium. At day 2 postinfection, supernatants of the infected cells were transferred to infect naïve Calu-3 and A549hACE2 for the second round. Fluorescence and phase contrast images for the infected cells are presented. (B) RT-PCR analysis of viral RNA. Extracellular RNAs from the second round of 
641

642

643

644

645

646

647

648

649

650

651

652

653

654

655

656

657

658

659

660

661

662

663

664

665

666

667

668

669

670

671

672

673

674

675

676

677

678

679

680

681

682

683

684

685

686

687

688

689

690

infection from (A) were harvested at day 2 post-infection and subjected to RT-PCR analysis of viral RNA.

Figure S3. No WT mNG SARS-CoV-2 production from the trans-complementation system. WT mNG SARS-CoV-2 and P10 $\triangle$ ORF3-E mNG virion (derived from 10 rounds of passaging of $\triangle$ ORF3-E mNG virion on Vero-ORF3-E cells) were used to infect Vero E6 cells for two rounds as described in Fig. 1G. (A) Fluorescence and phase contrast images of infected cells are presented for both the first and second rounds of infections. (B) RT-PCR analysis of viral RNA extracted from the culture fluids from the second-round infected cells.

Figure S4. Selection of $\triangle O R F 3-E$ mNG virion capable of inefficiently infecting Vero E6 cells for more than one round. Four independently selected P5 $\triangle \mathrm{ORF} 3-\mathrm{E}$ mNG virions (generated from five rounds of passaging $\triangle$ ORF3-E mNG virion on Vero-ORF3-E cells) were used to infect naïve Vero E6 cells for two rounds as described in Fig. 1G. The P5 $\triangle$ ORF3-E $\mathrm{mNG}$ virion-infected Vero E6 cells were analyzed for $\mathrm{mNG}$ signals $(\mathbf{A})$. The extracellular RNA from the second-round infected cells were examined for viral RNA by RT-PCR (B). Selection IV P5 $\triangle$ ORF3-E (S-IV-P5) mNG virion could infect Vero cells for multiple rounds. To remove the single-round virion from the multi-round virion in the S-IV-P5 stock, the S-IV-P5 stock was passaged on Vero E6 cells for two rounds, resulting in S-IV-P5-Vero-P2 virion capable of multiround infection. The replication kinetics of WT mNG SARS-CoV-2 and S-IV-P5-Vero-P2 mNG virion were compared on Vero E6 cells (C). The cells were inoculated at an MOI of 0.001. Limit of detection, L.O.D. Adaptive mutations were identified from the S-IV-P5-Vero-P2 mNG virion (D). The T130N mutation from the M protein was engineered into $\triangle$ ORF3-E mNG virion. The resulting $\triangle$ ORF3-E mNG M T130N virion was used to infect Vero E6 cells for two rounds. Fluorescence and phase contrast images of the infected cells are shown (E). Sequence alignment shows that the M proteins from SARS-CoV and SARS-CoV-2 share the same T130 residue (F). Red arrow indicates the T130 residue of SARS-CoV-2.

Figure S5. No improvement of viral replication of selection IV $\triangle$ ORF3-E (S-IV-P5) mNG virion after 10 rounds of culturing on Vero E6 cells. S-IV-P5 mNG virion was continuously passaged on Vero E6 cells for 10 rounds. The resulting P2 and P10 S-IV-P5 mNG virions (i.e., S-IV-P5-Vero-P2 and S-IV-P5-Vero-P10, respectively) were used to infect Vero E6 cells at an $\mathrm{MOI}$ of 0.001 . The mNG-positive cells $(\mathbf{A})$ and the growth kinetics of the S-IV-P5-Vero-P2 and S-IV-P5-Vero-P10 virions (B) were compared. We did not use the S-IV-P5-Vero-P1 virion in this experiment because the $\mathrm{P} 1$ stock retained some carryover virions derived from the Vero-ORF3E trans-complementation culture. Viral titers were analyzed by unpaired T-test. ns, P>0.05.

Figure S6. Safety characterization of S-IV-P5-Vero-P2 virion in hamsters. The weight change $(\mathbf{A})$ and disease $(\mathbf{B})$ of hamsters $(n=5)$ that were intranasally infected with $5,000 \mathrm{TCID}_{50}$ of S-IV-P5-Vero-P2 virion. A high-titer stock of S-IV-P5-Vero-P2 virion used for this experiment was prepared by amplifying the virion on Vero-ORF3-E cells.

Figure S7. Safety analysis of S-IV-P5-Vero-P2 virion in K18-hACE transgenic mice. Sevento nine-week-old K18-hACE2 mice were inoculated with S-IV-P5-Vero-P2 virion via the intranasal (I.N.) or intracranial (I.C.) route. Mouse body weight and survival were monitored for 14 days. (A) Mouse weight loss after I.N. infection. Mice were infected with 2,500 TCID $_{50}$ of SIV-P5-Vero-P2 virion $(n=4)$ or PBS mock $(n=4)$ via the I.N. route. The mean \pm standard deviations are indicated. (B) Mouse survival after I.N. infection. (C) Mouse weight loss after I.C. infection. Mouse were inoculated with 500 TCID50 of S-IV-P5-Vero-P2 virion $(n=4)$ via the I.C. route. The mean \pm standard deviations are indicated. (D) Mouse survival after I.C. infection. A 
bioRxiv preprint doi: https://doi.org/10.1101/2021.01.16.426970; this version posted January 19, 2021. The copyright holder for this preprint (which was not certified by peer review) is the author/funder. All rights reserved. No reuse allowed without permission.

691 high titer stock of S-IV-P5-Vero-P2 virion used for this experiment was prepared by amplifying 692 the virion on Vero-ORF3-E cells.

693 
694 Table S1. Comparison of neutralization titers between $\triangle$ ORF3-E mNG virion and 695 PRNT assays

696

697

\begin{tabular}{|c|c|c|}
\hline Serum ID & $\begin{array}{c}\Delta \text { ORF3-E virion- } \\
\text { NT }_{50}\end{array}$ & PRNT $_{50}$ \\
\hline 1 & $<20$ & $<20$ \\
\hline 2 & $<20$ & $<20$ \\
\hline 3 & 59 & 80 \\
\hline 4 & 81 & 80 \\
\hline 5 & 169 & 160 \\
\hline 6 & 225 & 200 \\
\hline 7 & 274 & 320 \\
\hline 8 & 353 & 320 \\
\hline 9 & 370 & 320 \\
\hline 10 & 392 & 320 \\
\hline 11 & 394 & 400 \\
\hline 12 & 568 & 320 \\
\hline 13 & 585 & 800 \\
\hline 14 & 666 & 400 \\
\hline 15 & 677 & 640 \\
\hline 16 & 744 & 320 \\
\hline 17 & 909 & 800 \\
\hline 18 & 925 & 640 \\
\hline 19 & 1196 & 800 \\
\hline 20 & 1789 & 1600 \\
\hline
\end{tabular}

698 
Table S2. Primers for plasmids construction and RT-PCR analysis

\begin{tabular}{|c|c|}
\hline Primer name & Sequences (5' to $\left.3^{\prime}\right)$ \\
\hline pcov-F56-F1 & $\begin{array}{l}\text { TATACGAAGTTATATTCGATGCGGCCGCGTCTCAGAGTGCTTTGGTTTAT } \\
\text { GATAATAAG }\end{array}$ \\
\hline pncov-R5 & TCGCACTAGAATAAACTCTGAACTC \\
\hline pncov-F6 & AGTTCAGAGTTTATTCTAGTGCGAATAATTGCACTTTTGAATATG \\
\hline pncov-R6 & ATGGCTAGTGTAACTAGCAAGAATACCAC \\
\hline pncov-F7 & GTATTCTTGCTAGTTACACTAGCCATCCTTACTGCGCTTCG \\
\hline pncov-R8 & AGGTCGACTCTAGAGGATCC \\
\hline cov-21115-F & CATTTGTGGGTTTATACAACAAAAG \\
\hline TRS2-S-R & GAAAAACAAACATTATCCGGTTAGTTGTTAACAAG \\
\hline TRS2-S-F & CTTGTTAACAACTAACCGGATAATGTTTGTTTTTC \\
\hline S-TRS2-M-R & $\begin{array}{l}\text { GAAAAACTAATATAATATTTAATCCGGTTATGTGTAATGTAATTTGACTCC } \\
\text { TTTGAGC }\end{array}$ \\
\hline TRS2-M-F & CCGGATTAAATATTATATTAGTTTTTCTG \\
\hline M-TRS2-R & GTAATAAGAAAGCGTCCGGGATGTAGCAACAGTG \\
\hline M-TRS2-F & CACTGTTGCTACATCCCGGACGCTTTCTTATTAC \\
\hline $\begin{array}{l}\text { ORF6-TRS2- } \\
\text { mNG-R }\end{array}$ & CTTTGCTCACCATATCCGGTTAATCAATCTCC \\
\hline $\begin{array}{l}\text { ORF6-TRS2- } \\
\text { mNG-F }\end{array}$ & GGAGATTGATTAACCGGATATGGTGAGCAAAG \\
\hline $\begin{array}{l}\text { ORF7-TRS2- } \\
\text { ORF8-R }\end{array}$ & CAAGAAATTTCATATCCGGTTAGGCGTGACAAG \\
\hline $\begin{array}{l}\text { ORF7-TRS2- } \\
\text { ORF8-F }\end{array}$ & CTTGTCACGCCTAACCGGATATGAAATTTCTTG \\
\hline $\begin{array}{l}\text { ORF8-TRS2- } \\
\text { N-R }\end{array}$ & $\begin{array}{l}\text { CATTATCAGACATTTTAGTTTATCCGGTTAGATGAAATCTAAAACAACACG } \\
\text { AACGTC }\end{array}$ \\
\hline TRS2-N-F & CCGGATAAACTAAAATGTCTGATAATGG \\
\hline cov-28501-R & GGTGTTAATTGGAACGCCTTGTCC \\
\hline M-T130N-F & CCATGGCACTATTCTGAACAGACCGCTTCTAGAAAG \\
\hline $\mathrm{M}-\mathrm{T} 130 \mathrm{~N}-\mathrm{R}$ & CTTTCTAGAAGCGGTCTGTTCAGAATAGTGCCATGG \\
\hline $\begin{array}{l}\text { 5ロUTR- } \\
\text { TRS2-F }\end{array}$ & GATCTGTTCTCTAACCGGATTTTAAAATCTGTGTG \\
\hline $\begin{array}{l}\text { 5ロUTR- } \\
\text { TRS2-R }\end{array}$ & CACACAGATTTTAAAATCCGGTTAGAGAACAGATC \\
\hline $\begin{array}{l}\text { EcoR1- } \\
\text { mCherry-F }\end{array}$ & $\begin{array}{l}\text { CACTTCCTACCCTCGTAAAGAATTCGCCACCATGGTGAGCAAGGGCGAG } \\
\text { GAG }\end{array}$ \\
\hline F2A-optE-R & GACACAAAAGAATACATTGGCCCAGGGTTGGACTCGAC \\
\hline F2A-optE-F & CCCTGGGCCAATGTATTCTTTTGTGTCTGAAG \\
\hline $\begin{array}{l}\text { EcoR1-Cov- } \\
\text { optE-R }\end{array}$ & GGGGAGGGAGAGGGGCGGGAATTCCTACACCAGCAGGTCGGGGACC \\
\hline $\begin{array}{l}\text { EcoR1- } \\
\text { IRES-F }\end{array}$ & TAGGAATTCCCGCCCCTCTCCCTCCCCCC \\
\hline $\begin{array}{l}\text { EMCV-IRES- } \\
\text { R }\end{array}$ & ATTATCATCGTGTTTTTCAAAGGAAAACCACG \\
\hline $\begin{array}{l}\text { IRES- } \\
\text { optORF3-F }\end{array}$ & GTTTTCCTTTGAAAAACACGATGATAATATGGACCTGTTCATGAGAATC \\
\hline BamH1-Cov- & CTCGCAGGGGAGGTGGTCTGGATCCCTCACAGAGGAACAGATGTGGTG \\
\hline
\end{tabular}


bioRxiv preprint doi: https://doi.org/10.1101/2021.01.16.426970; this version posted January 19, 2021. The copyright holder for this preprint (which was not certified by peer review) is the author/funder. All rights reserved. No reuse allowed without permission.

701

\begin{tabular}{|l|l|}
\hline optORF3-R & G \\
\hline CoV-T7-N-F & ACTGTAATACGACTCACTATAGGATGTCTGATAATGGACCCCAAAATC \\
\hline polyT-N-R & $\left(\right.$ T) ${ }_{37}$ AGGCCTGAGTTGAGTCAGCAC \\
\hline CoV19-N2-F & TTACAAACATTGGCCGCAAA \\
\hline CoV19-N2-R & GCGCGACATTCCGAAGAA \\
\hline
\end{tabular}




\section{Figure 1}

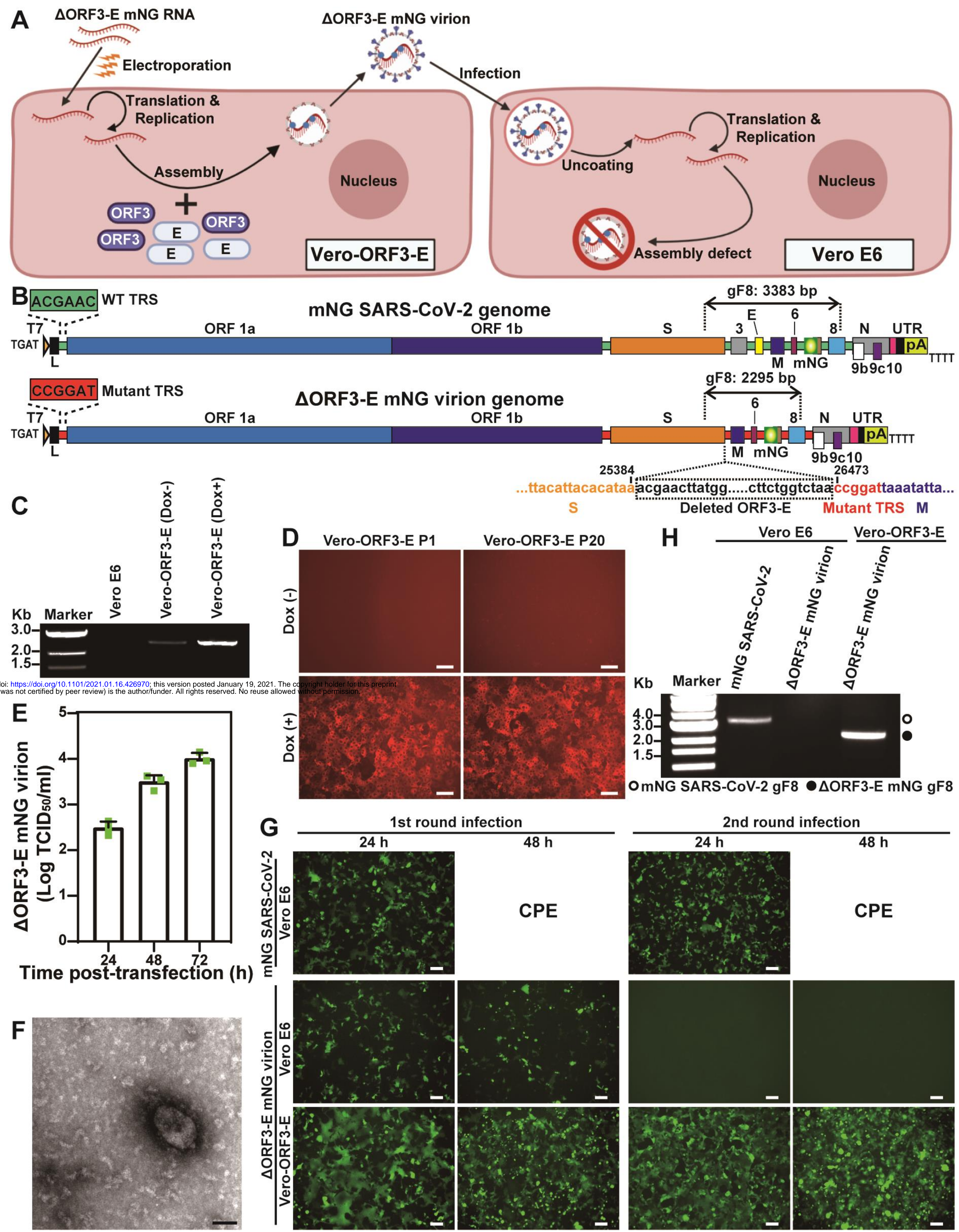


Figure 2
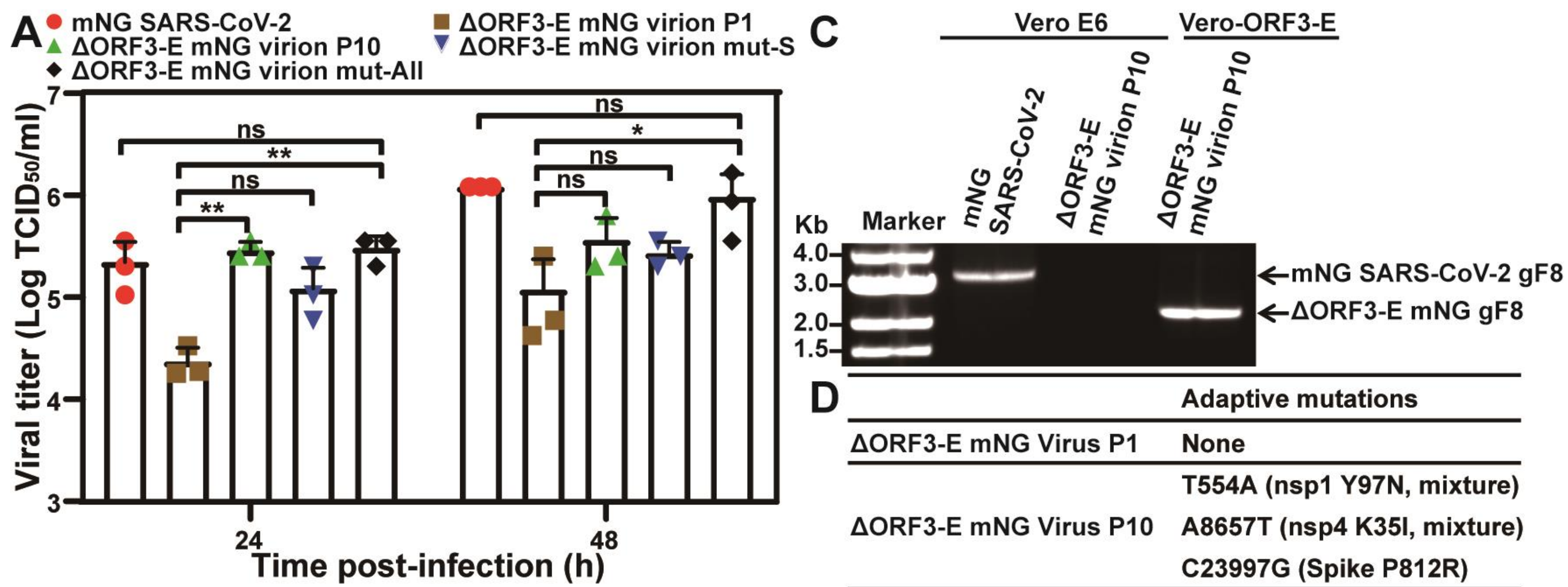

\section{B}

$\triangle$ ORF3-E

$\triangle$ ORF3-E mNG virion P10
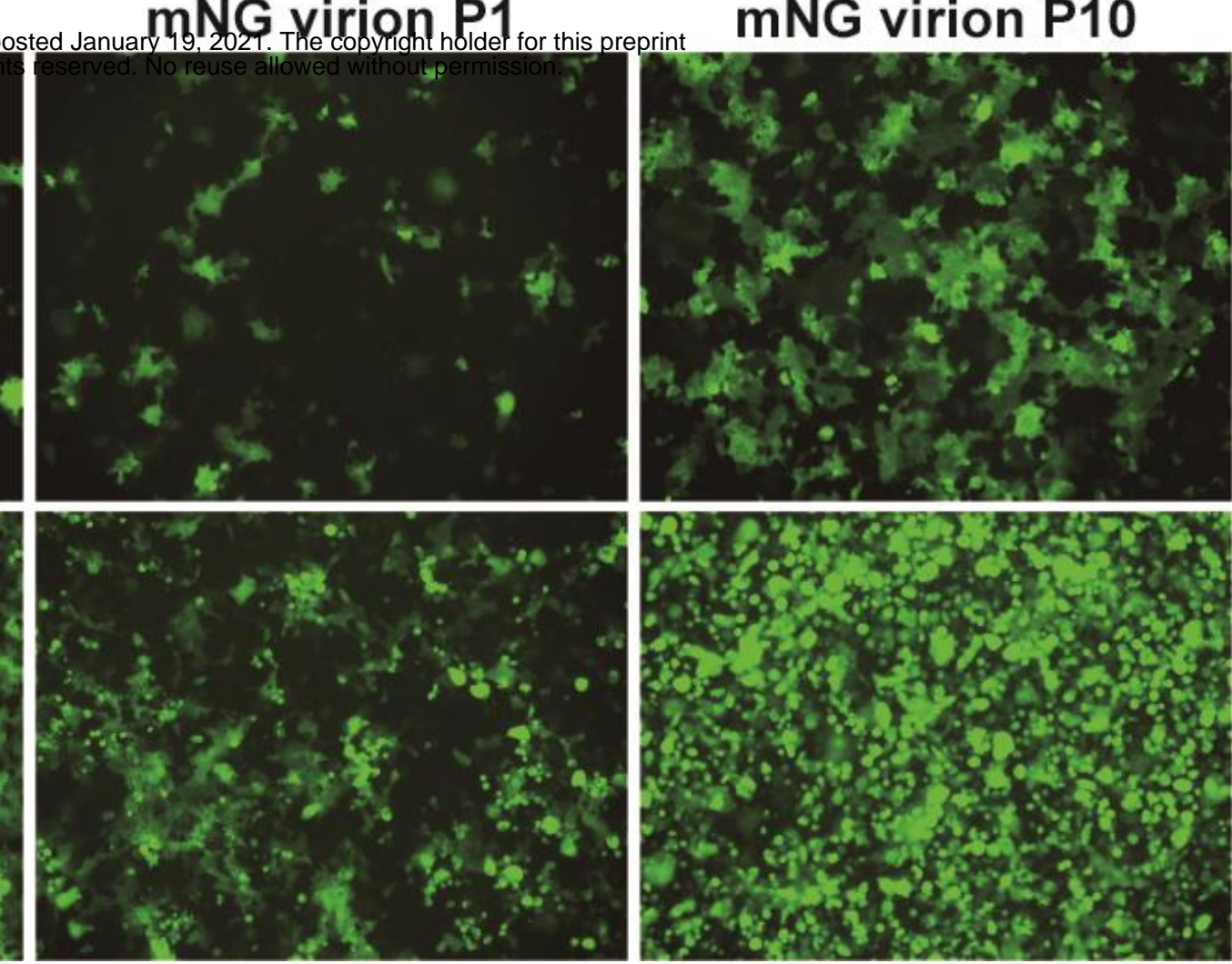
mNG virion mut-S $\triangle$ ORF3-E

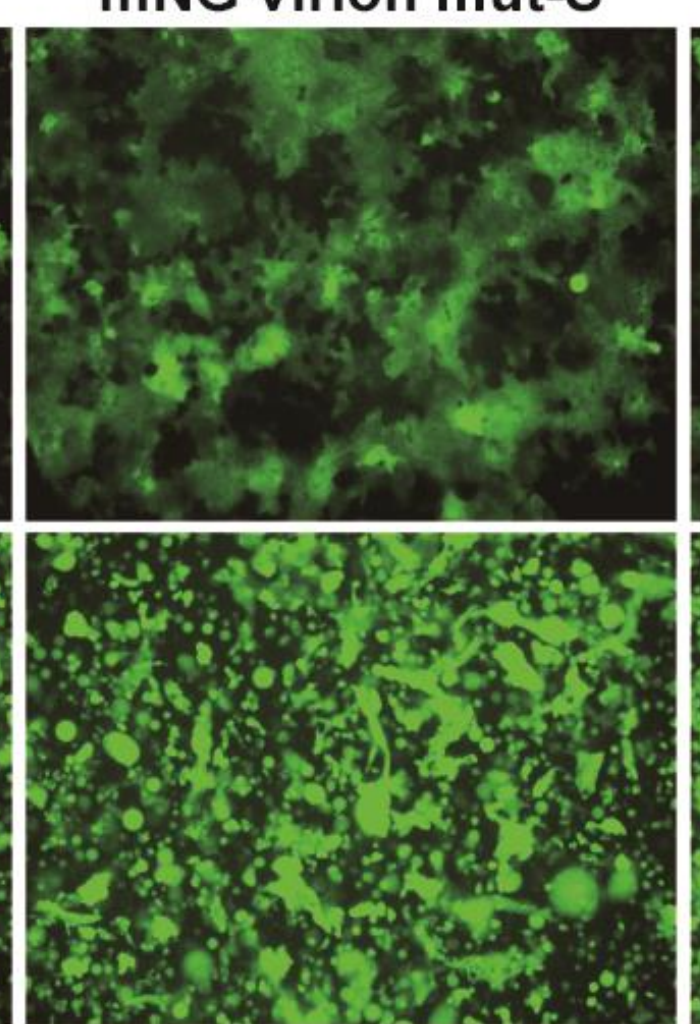

mNG virion mut-All

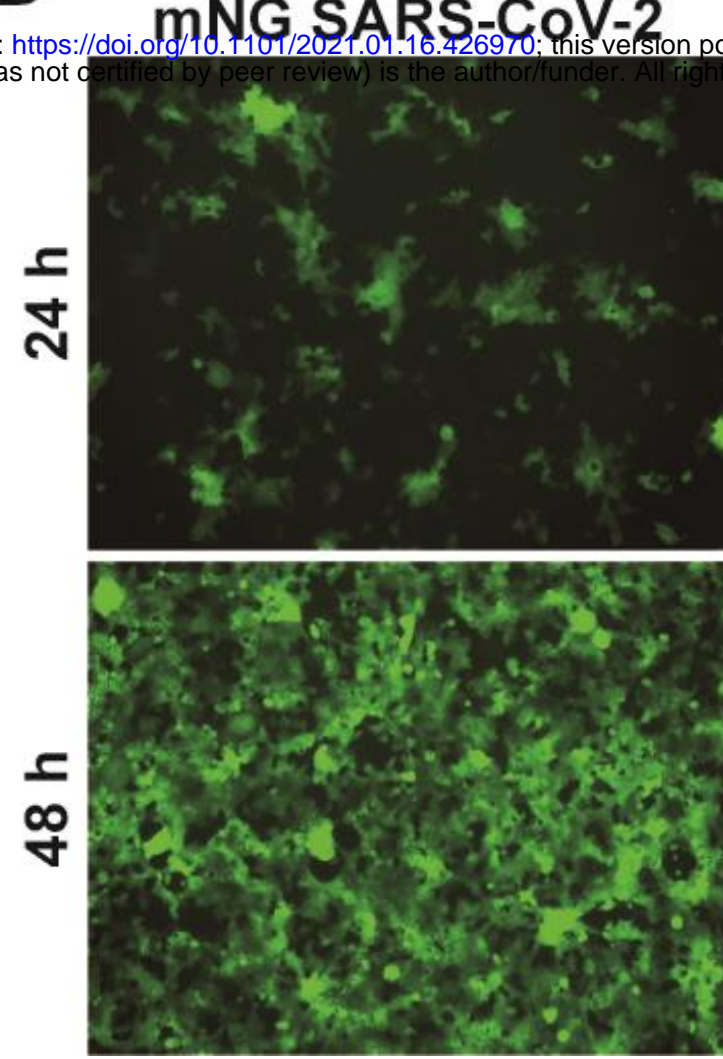



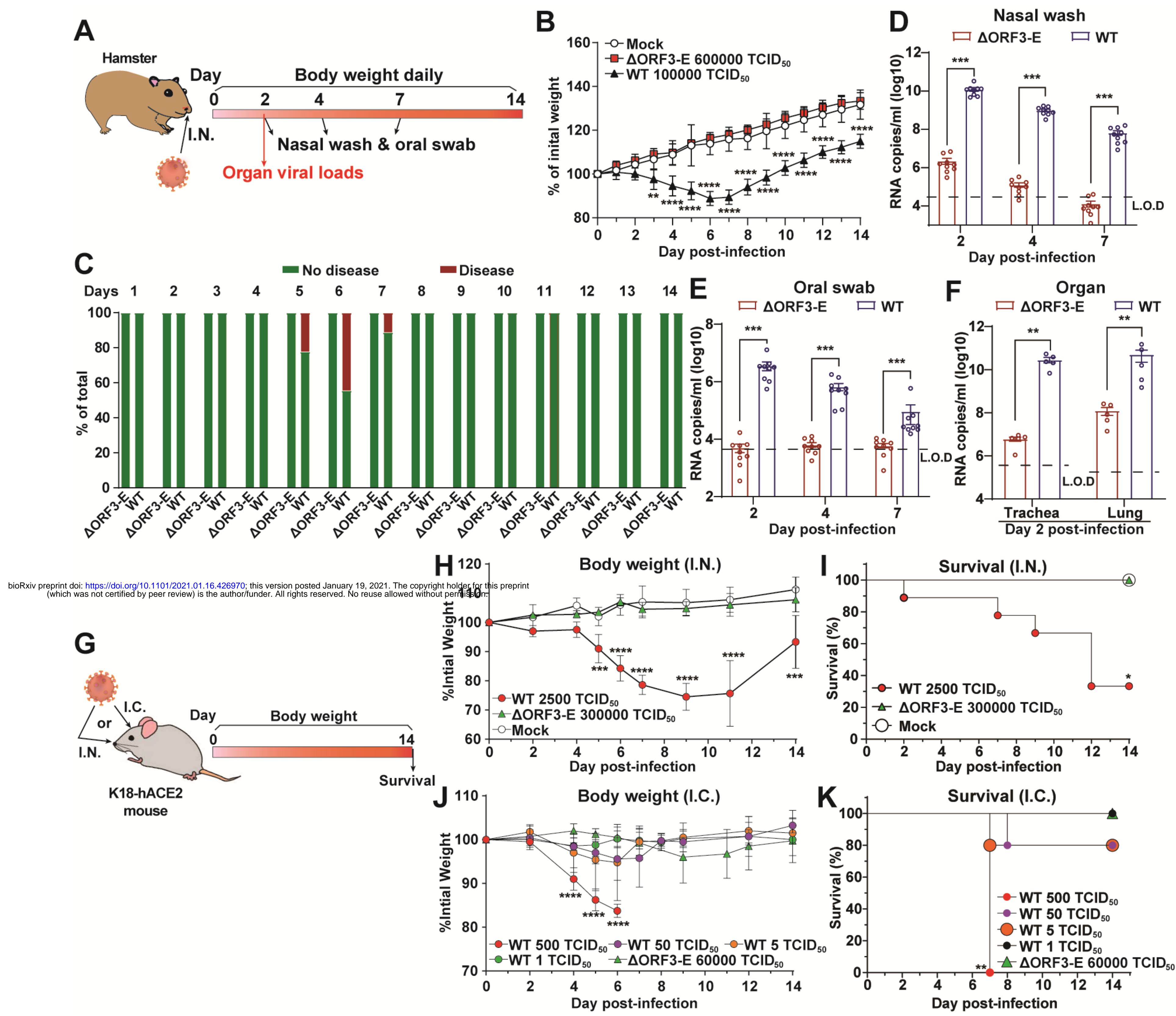
Figure 4
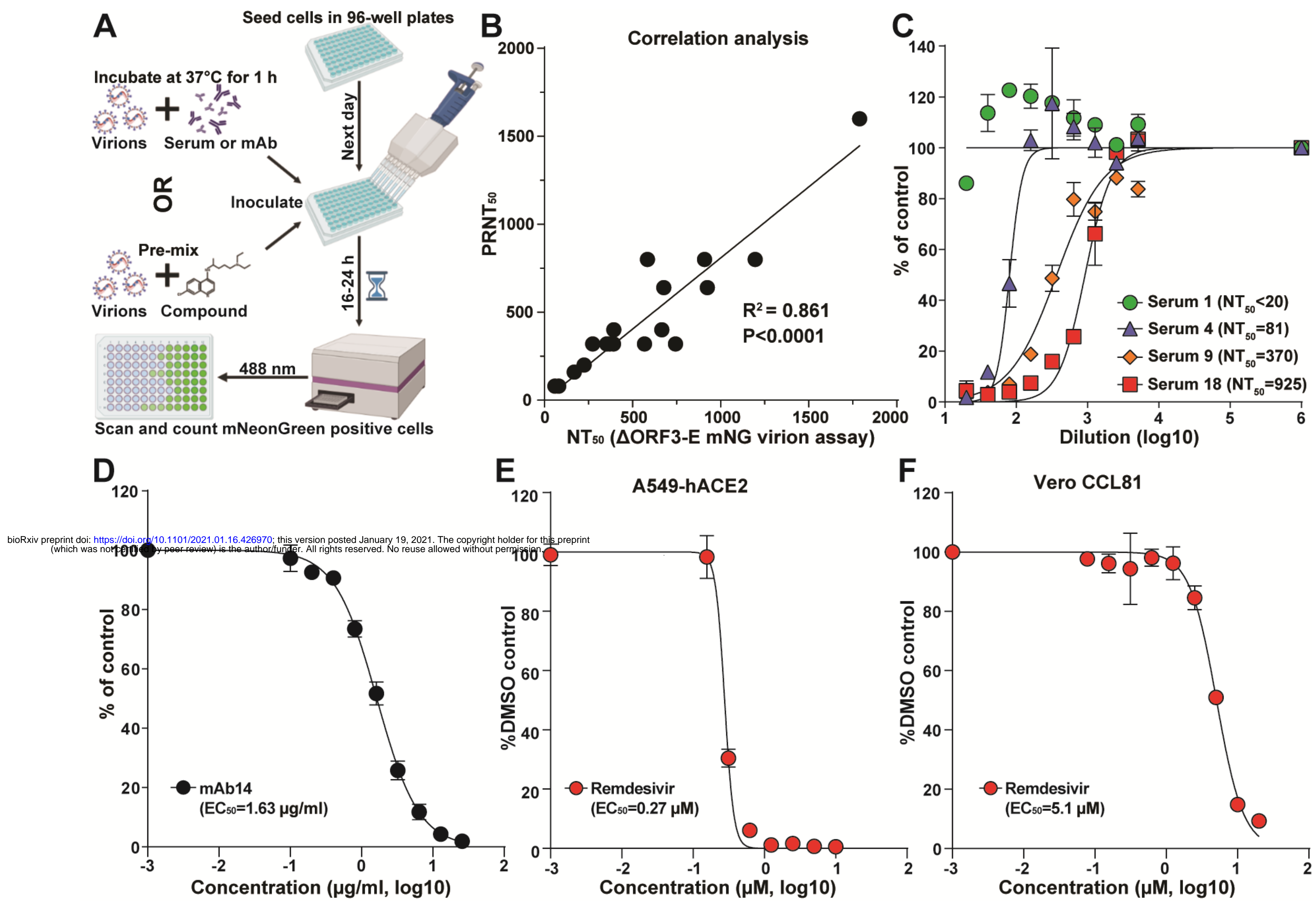
Figure S1
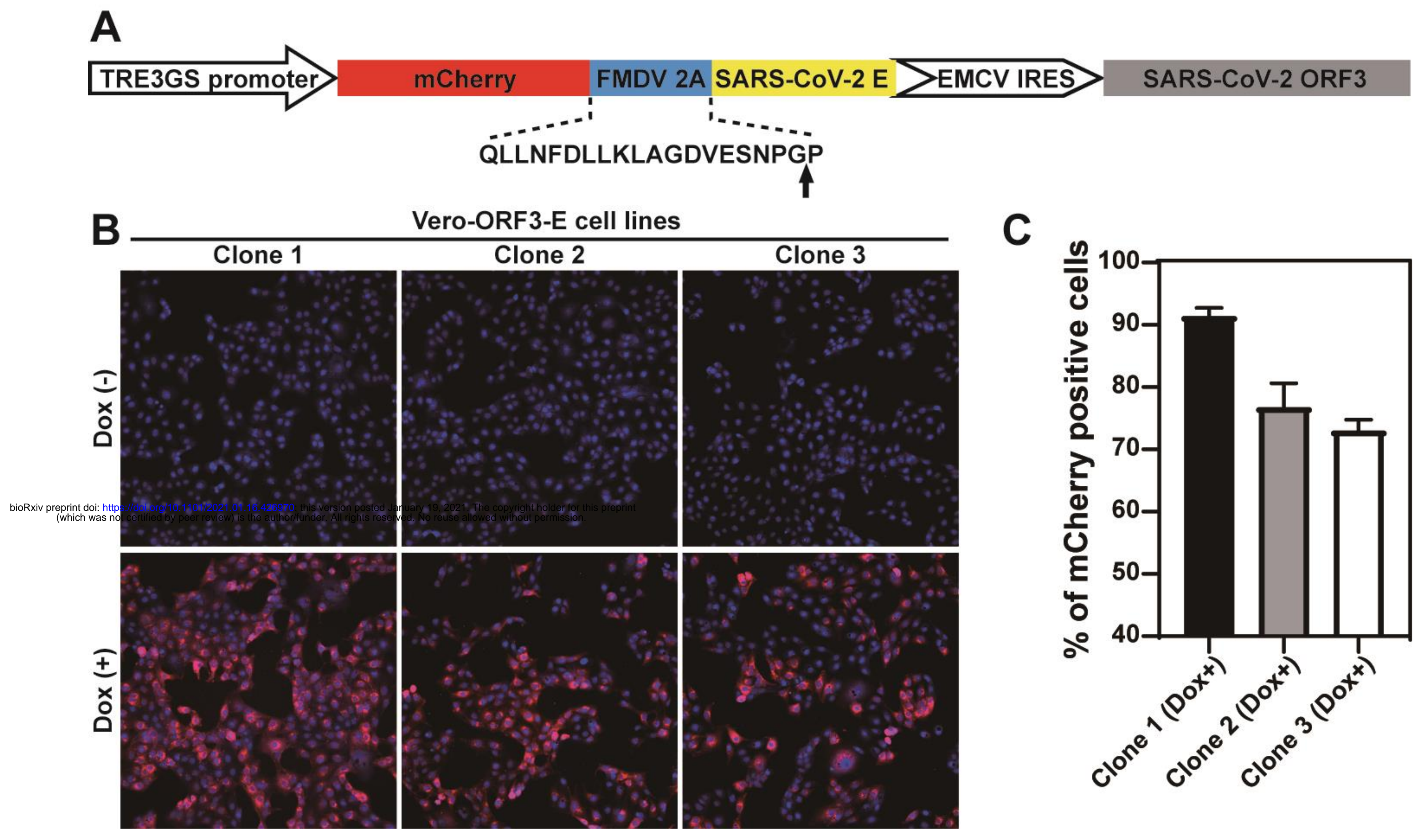
Figure S2
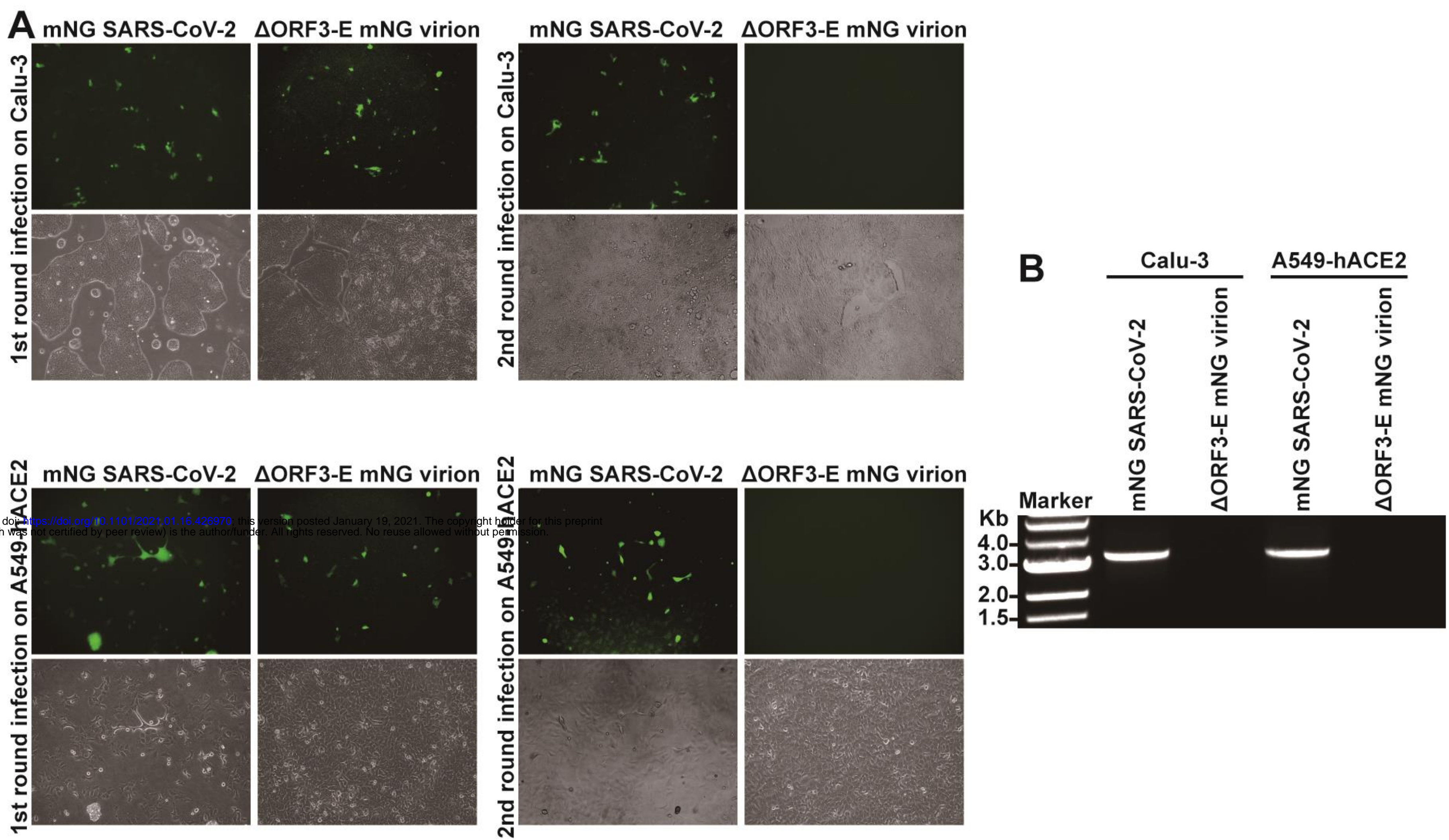
Figure S3

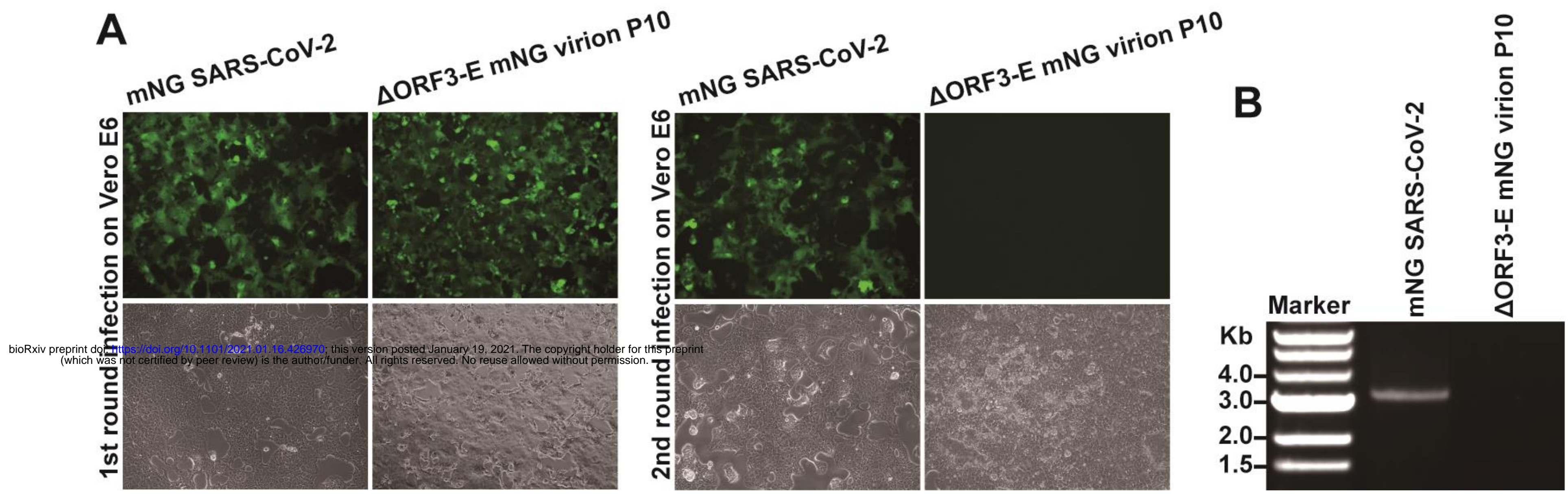




\section{Figure S4}

A
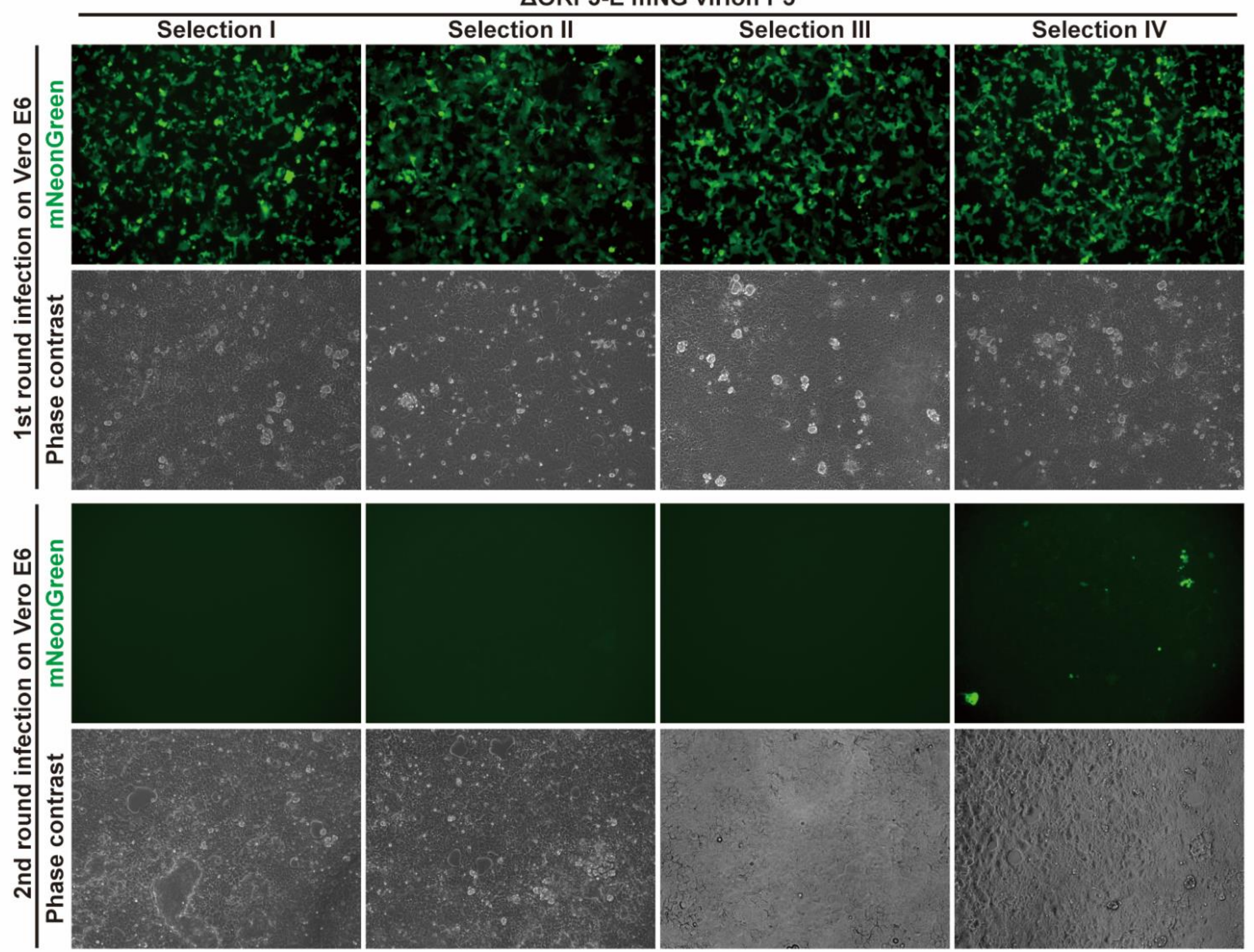

B

$\triangle O R F 3-E$ mNG virion P5

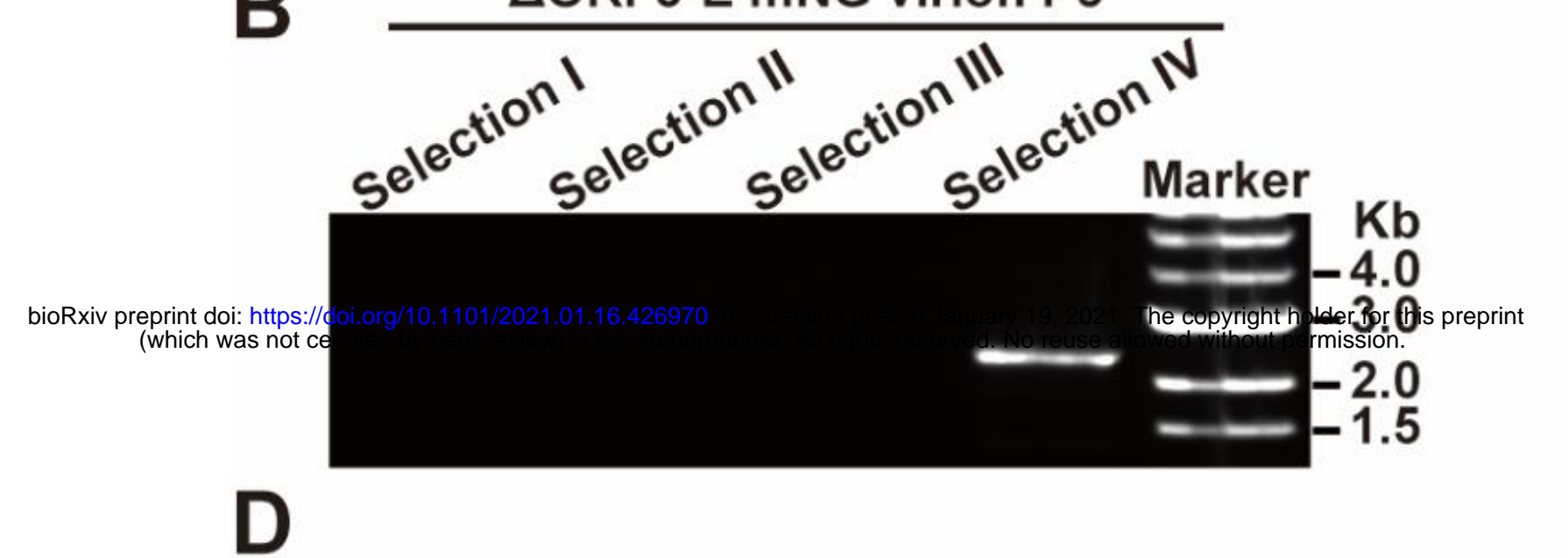

D

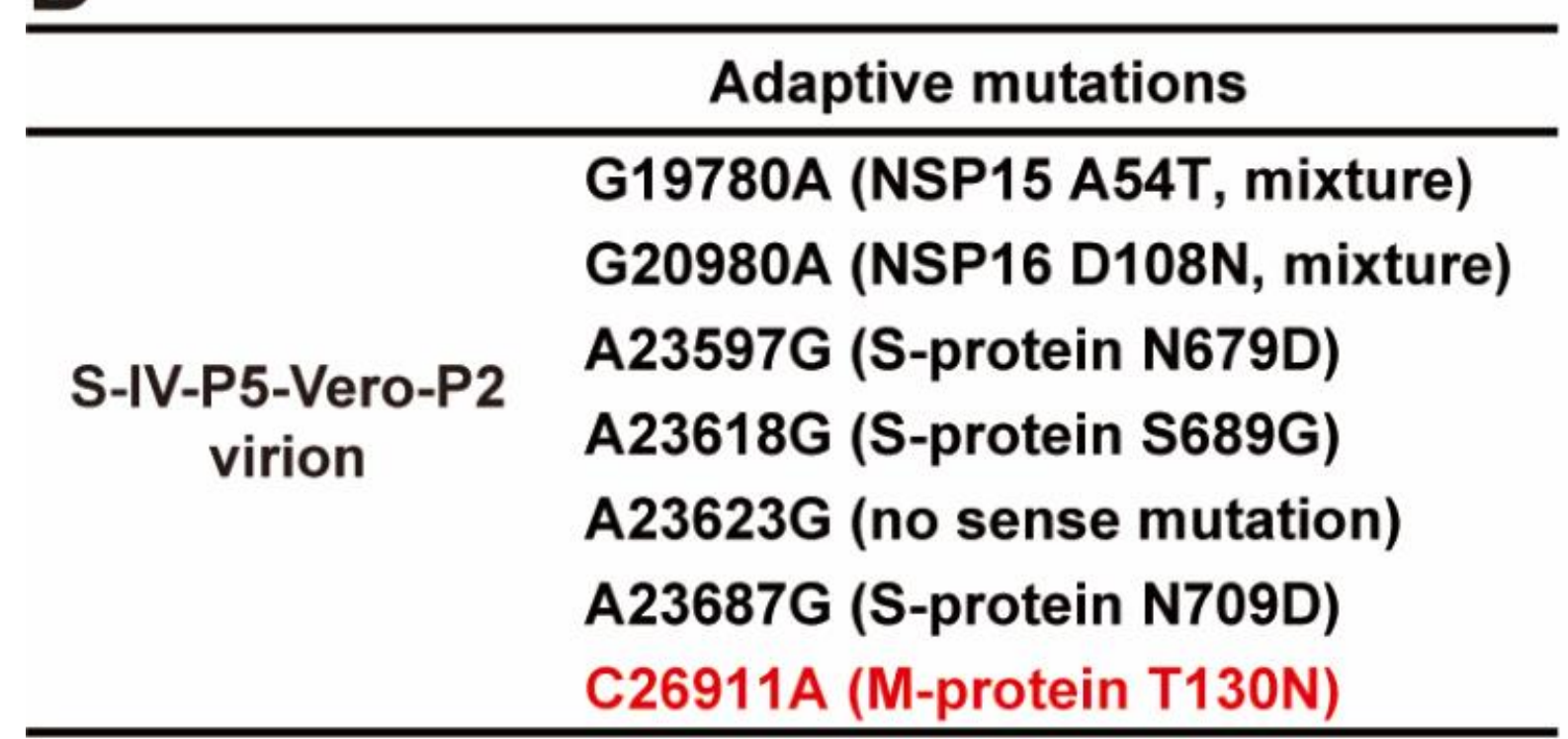

C mNG SARS-CoV-2

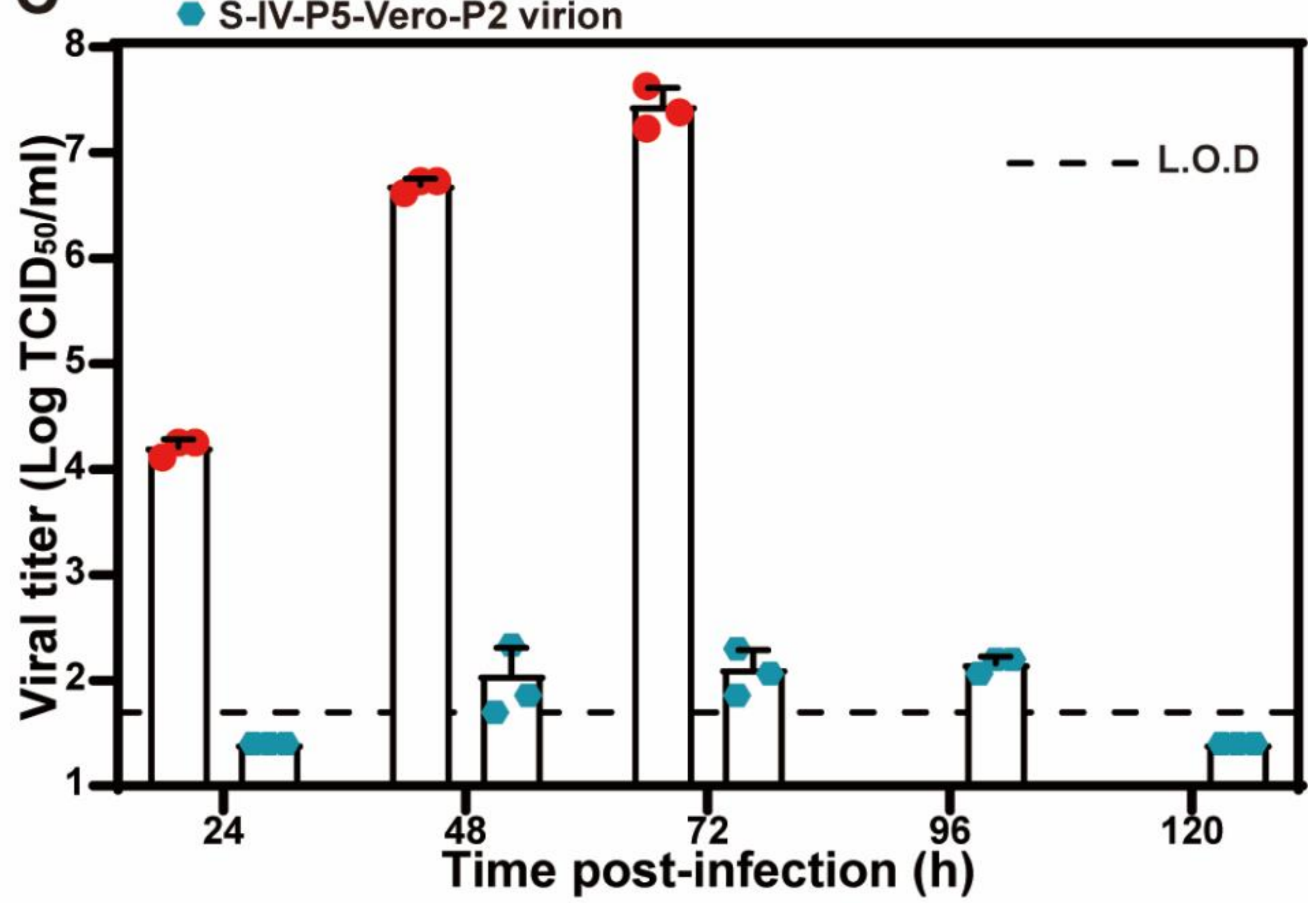

E

$\triangle$ ORF3-E mNG M T130N virion
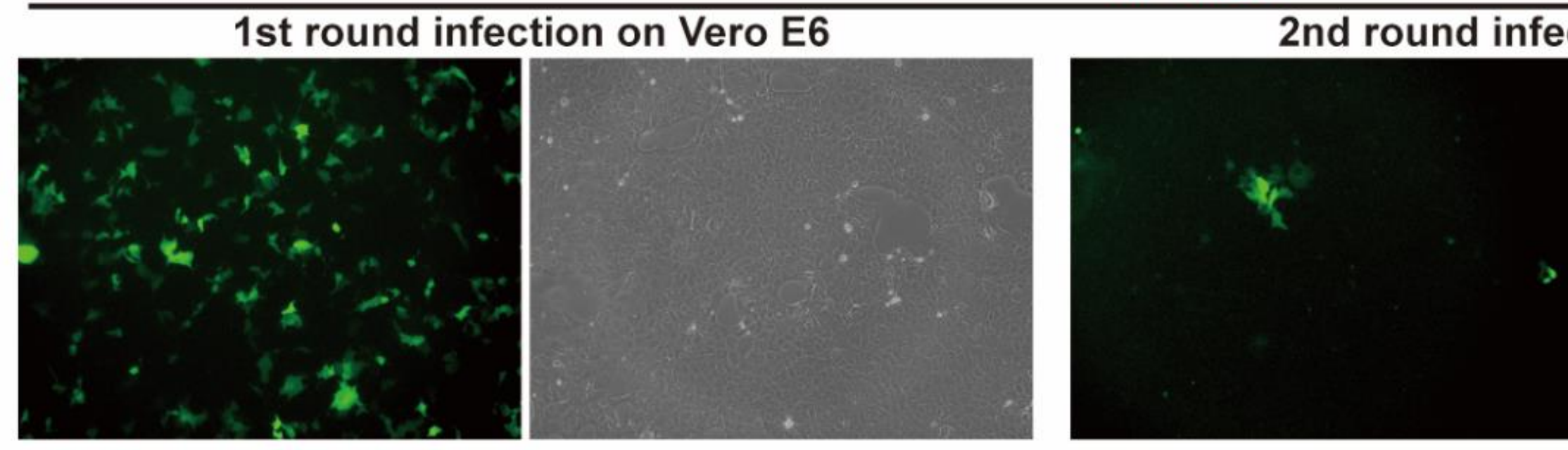

F

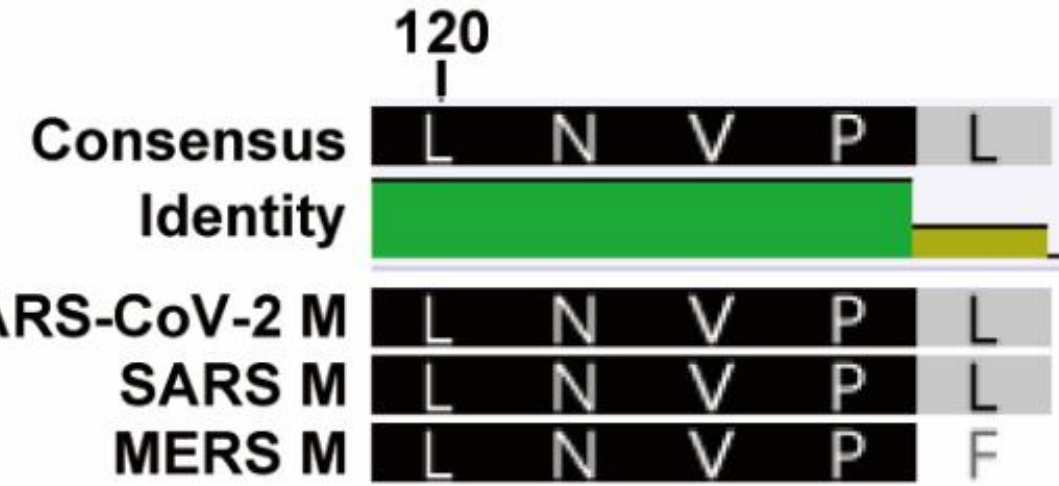
MERS M

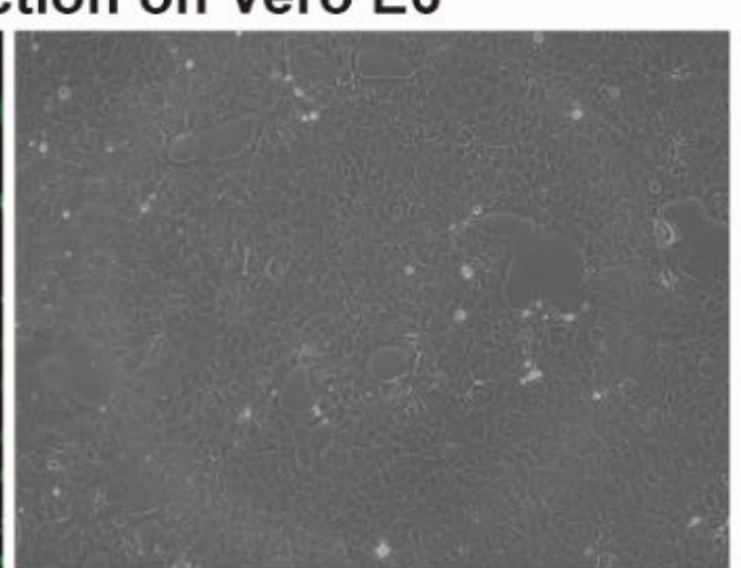

130

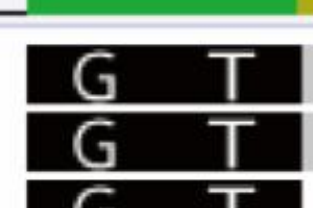

130

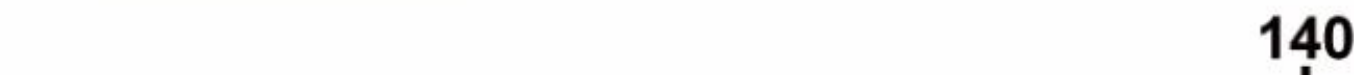


Figure S5
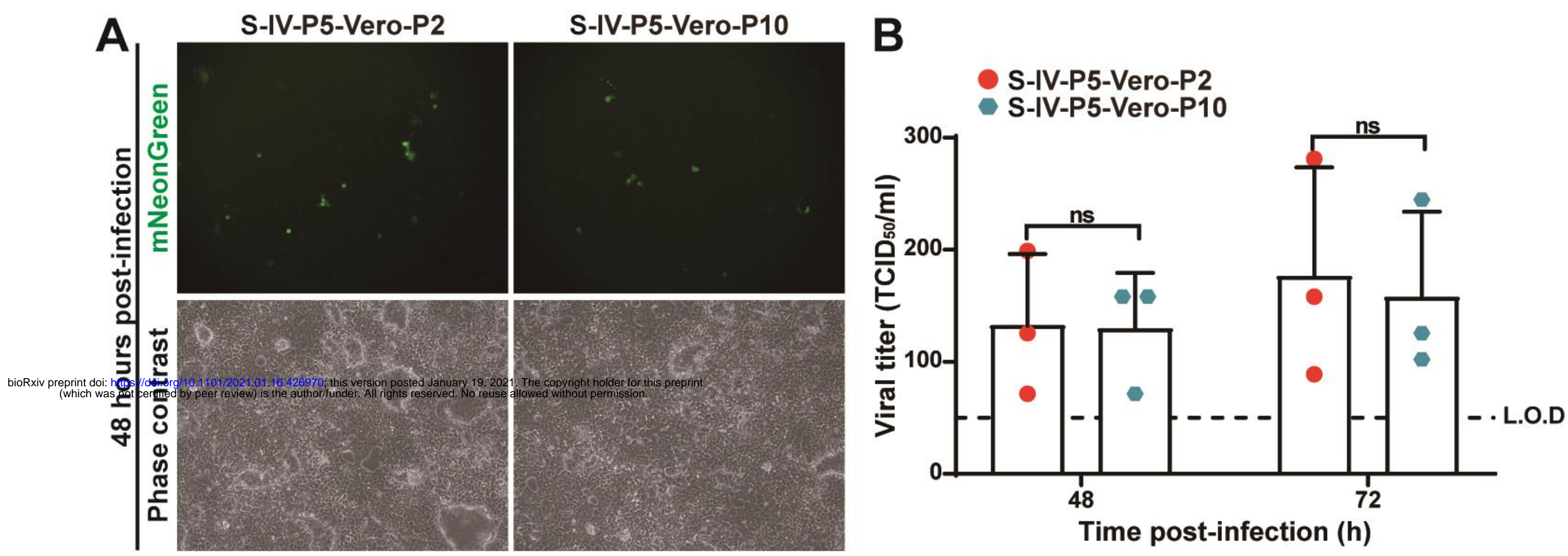
Figure S6

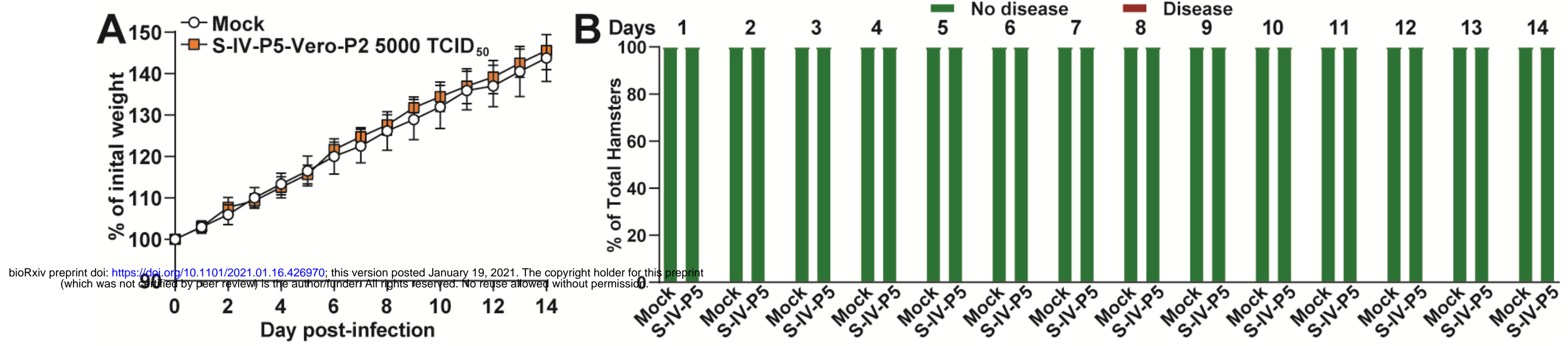


Figure S7

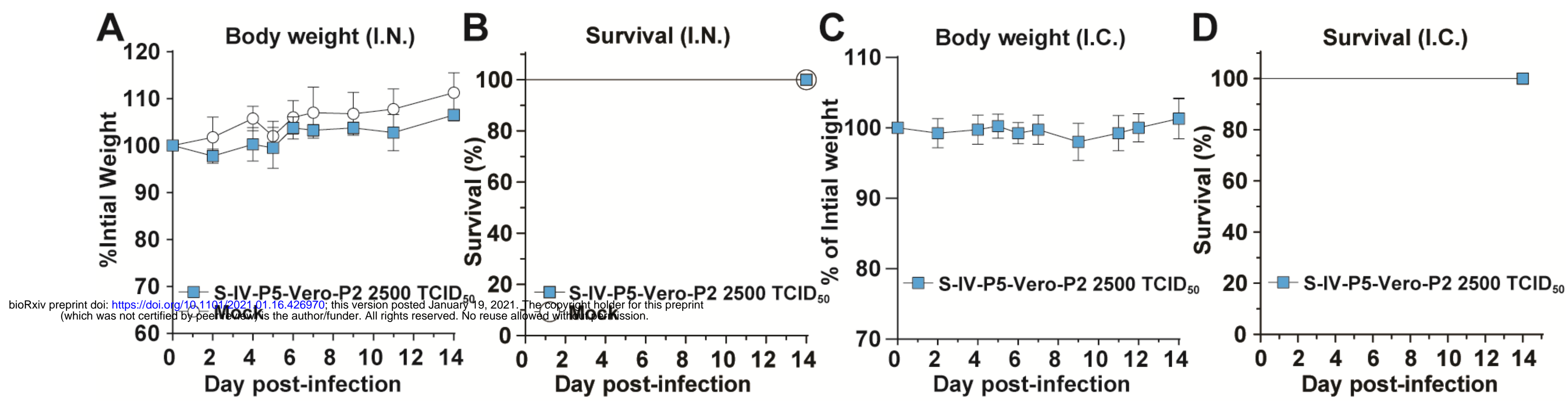

